\title{
The Correlation of Non-Destructive Measurements and Toughness Changes in Adhesive Joints During Environmental Attack
}

\author{
K.VINE, P. CAWLEY and A.J. KINLOCH \\ Department of Mechanical Engineering \\ Imperial College of Science, Technology and Medicine \\ Exhibition Road, London SW7 2BX, UK
}

(Received 2 June 2001; revised and accepted 1 August 2001.)

Normal-incidence ultrasonic scans have been conducted on two- and three-layer adhesive-joint specimens exposed to water at $50^{\circ} \mathrm{C}$ for periods of up to 18 months. The joints consisted of aluminium-alloy adherends which were subjected to one of four different surface pretreatments prior to being coated (for the two-layer specimens) or bonded (for the three-layer specimens) with an epoxy polymer. The four commonly used pretreatments which were investigated were a grit-blast, a chromic-acid etch (CAE), a chromic-acid anodise (CAA) and a phosphoric-acid anodise (PAA). Techniques have been developed to measure the fracture toughness, $G_{c}$, of the specimens before and after water exposure such that fracture toughness maps could be ascertained, where the measured values of $G_{c}$ may be assigned to spatially discrete positions within the specimens. The relative performance of the different pretreatments used in the present work followed that expected from the literature: the chromic-acid anodised (CAA) surface pretreatment giving the most durable two- and three-layer specimens and the grit-blasting pretreatment giving the least durable. In the two-layer specimens, the ultrasonic inspections detected two main types of defects: corrosion-driven edge-disbonds and micro-defects. The edge-disbonding mechanism usually started at an unsealed flush edge, and was initiated by a region of corrosion which developed on the edge of the specimen and which undercut the epoxy layer. Edge-disbonds were easily and accurately detected ultrasonically. Micro-defects were detected in regions remote from the edges and these small-scale, isolated defects took several forms. In the case of the three-layer joint specimens, only edge-disbonds could be detected ultrasonically. Nevertheless, in some of the three-layer joints which were attacked and weakened by ingressing water, the failure surfaces suggested that micro-defects were present. For both the two- and the three-layer specimens, the results from the ultrasonic scans have been correlated with the values of the fracture toughness of the specimens, before and after water exposure. Whilst the ultrasonic scans detected the presence of micro-defects in the two-layer specimens, which appeared to correlate with the extent of interphase toughness loss upon water exposure, the scans clearly failed to detect any changes in the interphase regions which would indicate the general loss of interphase toughness seen with the three-layer grit-blast and PAA specimens. 


\section{INTRODUCTION}

Adhesively-bonded aluminium-alloy structures are extensively used in the aerospace and other industries. Adhesive bonding gives a more uniform stress distribution than can be achieved with mechanical fasteners such as rivets, and avoids the problems associated with the welding of aluminium alloys. However, careful surface preparation of the adherends must be undertaken in order to ensure the long-term durability of adhesive joints between aluminium-alloy adherends, particularly when they are exposed to hot, wet environments [1].

A variety of surface preparation procedures are commonly applied to the aluminium-alloy surface prior to bonding [2]. A chromic-acid etch (CAE) followed by phosphoric-acid anodisation (PAA) is commonly used in the USA, while a CAE etch followed by chromic-acid anodisation (CAA) is the standard European aerospace treatment. These treatments produce characteristic oxide structures on the aluminium-alloy surface. The oxide layer produced by the CAE etch alone is about $0.05 \mu \mathrm{m}$ to $0.07 \mu \mathrm{m}$ thick, while those generated by the PAA and CAA treatments, when carried out to the relevant standards, are approximately $0.5 \mu \mathrm{m}$ and $3.5 \mu \mathrm{m}$ thick respectively [2].

The morphology of the oxides produced by the anodisation processes resembles a porous honeycomb structure. Further discussion of the oxide structures produced by the different surface preparation procedures may be found in O'Sullivan and Wood [3], Thompson et al. [4], Thompson and Wood [5] and $\mathrm{Xu}$ et al. [6]. The adhesive (or a primer, as is typically used) often flows into the pores of the oxide structure during the curing process, so forming a 'micro-composite' 'interphase' region [7]. The extent of this penetration is a function of the pore size and the viscosity of the adhesive (or primer) used.

The interphase between the bulk adhesive and the bulk adherend formed by the oxide is a major determinant of the susceptibility of the joint to environmental attack. There is therefore a need to monitor the properties of the interphase region both immediately after a joint is produced and during service. However, this task is very difficult due to the small thickness of the interphase region produced with the standard PAA and CAA processes compared with a typical adhesive layer thickness of $100 \mu \mathrm{m}$ or more, and an adherend thickness which is generally over $1000 \mu \mathrm{m}$. Once the joint has been made, the interphase is not accessible so it must be interrogated via the adherend.

There has been considerable interest in this problem for many years and a review of early work in the field is given by Thompson and Thompson [8]. Ultrasonic methods have generally been 
regarded as the potentially most useful and the bulk of the research effort has been concentrated in this field. Some promising results have been obtained but the capability of the various possible testing techniques has yet to be fully defined and no technique is ready for industrial implementation. Four basic categories of ultrasonic technique have been investigated [9]. These are ultrasonic reflection coefficient measurements at normal- and/or oblique- incidence [10-13]; Lamb wave measurements [14-16]; the propagation of true guided waves in the adhesive layer [17-20]; and the measurement of the zeroes of the reflection coefficient from the adhesive layer, which is related to the propagation of leaky guided waves along the layer [21-24]. Most researchers have assumed (implicitly or explicitly) that changes in the interphase due to environmental attack are likely to occur relatively uniformly over the area interrogated by the transducer. Calculations based on likely interphase properties [9] indicated that the measurement of oblique-incidence reflection coefficients is likely to be the most promising technique in practice, since its sensitivity to the interphase characteristics is at least as good as that of the other methods and the reflection coefficients are relatively insensitive to small changes in the bulk adherend and adhesive properties. However, the test involves the accurate monitoring of the amplitude of the reflection from an embedded interface which is not a simple task, particularly at oblique incidence. This had led other researchers, notably Rokhlin and co-workers [22], to favour the measurement of reflection coefficient zeroes, which involves frequency, rather than amplitude, measurements.

This paper reports a study of the changes in the ultrasonic reflection characteristics of a series of bonded joints with different pretreatments during exposure to water at $50^{\circ} \mathrm{C}$. The changes observed in the non-destructive tests are then correlated with the toughness of the joint measured using a double-cantilever beam (DCB) test.

\section{SPECIMENS and EXPERIMENTAL TECHNIQUES}

\subsection{Specimens}

Two main types of specimen were used, a two-layer and a three-layer specimen, as shown in Fig. 1. The epoxy adhesive used was an unmodified two-part epoxy from Ciba Geigy, 'AY103' cured with an amine hardener, 'HY 951'. The curing cycle was 48 hours at room temperatures, except where specified. The aluminium alloy used was an aerospace grade, 'L157'.

Two-layer specimens similar to that shown in Fig. 1 were employed in earlier work by Cawley et al. [13], and also by Jackson et al. [25] and Spelt et al. [26]. The two-layer specimen was chosen for several reasons. Key amongst these was that an exposed epoxy layer would allow water to 
diffuse through the epoxy to the interface in a uniform manner. Also, when a $2 \mathrm{~mm}$ thick layer is used, the epoxy layer would reach saturation over the course of some months. An epoxy thickness of $2 \mathrm{~mm}$ also results in a good separation between ultrasonic echoes. A three-layer specimen will absorb water in a non-uniform manner and, with a large area of overlap, will take many years to reach saturation. For both types of specimen, variations in the edge conditions were also investigated, with two of the edges of the specimens being sealed with a marine sealant, and two being left unsealed. Further, two edges were produced with a flush epoxy-aluminum edge, with the remaining two having an overlap between the edge of the aluminum and the epoxy. The recessed edges, giving rise to the overlap, would allow any degradation advancing from the joint edge to be detected immediately, whereas the flush edges could not be inspected ultrasonically to the edge of the epoxy layer, due to the finite size of the ultrasonic probe. Four common pretreatments were investigated: grit-blast, chromic-acid etch (CAE), chromic-acid anodising (CAA) and phosphoricacid anodising (PAA). The three-layer specimen maintained the same geometry as the two-layer specimen, with the exception of the second adherend being present and the thickness of the epoxy layer being reduced to $0.2 \mathrm{~mm}$, which is more realistic of a structural adhesive joint. Both specimen types were immersed in water at $50^{\circ} \mathrm{C}$ and inspected at intervals. The top aluminium-alloy surfaces were protected with a rubber sealant while the specimens were immersed. This prevented gross corrosion of the aluminium alloy, so leaving a smooth surface when the sealant was removed prior to ultrasonic testing. (Fresh sealant was re-applied before re-immersing the specimens in the water at $50^{\circ} \mathrm{C}$.)

\subsection{Ultrasonic techniques}

Two ultrasonic techniques were used for the inspection of all of the specimens: normal- and oblique-incidence scanning. Normal-incidence pulse echo scans were performed using a $50 \mathrm{MHz}$ focused transducer. The centre frequency of the received signal was maintained above $50 \mathrm{MHz}$ by using a very short water path. This produced scans with a spot size of approximately $0.1 \mathrm{~mm}$. Oblique incidence scans were also performed. These used a pair of $20 \mathrm{MHz}$ focused transducers, inclined at $16.8^{\circ}$ in water, which produced a shear wave in the aluminium alloy at $37^{\circ}$. It was found that the normal-incidence scans gave better resolution of small defects than the oblique-incidence measurements and the oblique-incidence scans provided no indications of other changes that were not apparent on the normal-incidence scans. Therefore all the ultrasonic results in this paper are from the normal-incidence measurements; the difference between the normal- and obliqueincidence measurements and the reasons for the greater sensitivity of the normal-incidence 
measurements, in contrast to predictions made in the previous literature, are discussed in detail in another paper [27].

\subsection{Mechanical testing}

Mechanical tests were performed on the two- or three-layer specimens after they had been immersed in hot water for various lengths of time. A mechanical test that would give spatially discrete information about the toughness of the specimens was desirable. It was decided that a double-cantilever beam (DCB) test would be a useful test vehicle for such studies, especially if stable crack growth along the specimen was observed. For all the DCB tests a constant rate of displacement of $0.5 \mathrm{~mm} / \mathrm{min}$ was employed. The load was applied to the end of the specimen, and the load and crack length were measured. Knowing the geometry of the joint and the load at a given crack length allowed the adhesive fracture energy, $G_{c}$, to be ascertained [28, 29]. For both the twoand three-layer specimens the results were then displayed in a similar fashion to a 'C-scan', with a grey-level square representing the toughness at a given point on the specimen.

In order to prepare the DCB tests, the two- or three-layer specimens were cut into $10 \mathrm{~mm}$ wide strips. In the case of the two-layer specimens, each strip was bonded to a stiff aluminium-alloy base and an end block was glued to one end of the aluminium-alloy adherend. With the specimen bonded to the stiff base the failure would tend to propagate at, or very close to the aluminium-alloy/epoxy interface [29]. For the three-layer specimens a similar approach was used. In this case, end blocks were glued to both aluminium-alloy adherends at one end of the specimen. The failure of these specimens was generally through the epoxy layer, except in the cases were there had been degradation of the interface, when the crack would propagate along, or close to, the interface. Finally, it should be noted that the first two-layer grit-blasted specimen, which was studied extensively using the ultrasonic test techniques described previously, was also employed to develop these mechanical test methods. However, undoubtedly due to it being used as the development test specimen, a relatively large scatter was recorded for the $G_{c}$ values. Hence, a duplicate test specimen was examined and the values of the toughness for the two-layer grit-blasted specimen shown in Table 1 are the results from the duplicate test specimen, which was exposed for a total of 145 days in water at $50^{\circ} \mathrm{C}$. However, it should be noted that the results from both specimens were in agreement, albeit bearing in mind the relatively large scatter that was recorded in the values of $G_{c}$ for the first, development test, specimen. 


\section{RESULTS: TWO-LAYER SPECIMENS}

\subsection{Introduction}

Table 1 shows a summary of the results obtained from the two-layer specimens. It was found from the ultrasonic inspections that were periodically conducted that edge-disbonds initiated at the edges of all the two-layer specimens and after initiation their rate of growth depended on the durability imparted by the chosen surface pre-treatment. Isolated micro-defects were seen in regions remote from the edges and again their number and growth depended on the durability imparted by the chosen surface pre-treatment. These two types of defect that were detected are discussed in more detail below.

After the final ultrasonic tests had been completed, the toughness, $G_{c}$, of the specimens was measured as described above. In all cases the locus of joint failure was visually assessed as being along the epoxy-adhesive/aluminium-oxide interface, and a similar observation was typically recorded for the unexposed 'control' specimens. It should be noted that the calculation of the average toughness for the degraded specimen discounted the regions of the edge-disbonds, which had already failed prior to the mechanical tests and so possessed zero toughness. Thus, for all the specimens examined any loss in interphase toughness is related to the areas which were still bonded, albeit with the presence of some micro-defects. However, the toughness data for the CAA specimens shown in Table 1 reveals an increase for the specimen tested after environmental exposure, compared to the 'control' which was not exposed. This was considered to be due to water being rapidly absorbed by, and plasticising, the epoxy and so increasing the toughness of the epoxy layer. From the results shown in Table 1, the detection of micro-defects is clearly a strong indicator that the interphase of the specimen is being attacked by the ingressing water molecules and, indeed, the presence of such defects correlates in a semi-quantitative manner to the loss of interphase toughness. Hence, the discussions below concentrate on the detection and interpretation of these micro-defects.

\subsection{Grit-blast specimens}

Considering the ultrasonic tests, Fig. 2 shows the results obtained from the normal-incidence scans of a two-layer grit-blast specimen. The figure label details how many days the specimen had been immersed in water at $50^{\circ} \mathrm{C}$ for each of the scan images presented. The scale at the top of the figure shows the signal amplitude associated with the grey scale of each image. It is shown as a percentage of the maximum measurable by the digitiser; signal amplitudes above the upper limit are shown in black, and those below the lower limit are shown in white. 
In the various normal-incidence scans that comprise Fig. 2 several different features may be identified. Firstly, there is an area of white speckle which is due to surface roughness. The effect of roughness can also be seen in the reflections from the disbonded regions of the sample; as time progresses, corrosion roughens the aluminium-alloy surface, and the reflected signal amplitude drops. Secondly, there are blurred light-shaded lines visible. These arise due to scratches on the top al uminium-alloy surface, produced during removal of the protective rubbery-seal ant coating prior to scanning. Thirdly, an obvious relevant defect to develop in the specimen with increasing exposure time to water at $50^{\circ} \mathrm{C}$ is the growth of corrosion-driven disbonds from the unseal ed edges, and these advance rapidly toward the centre of the specimen. Fourthly, another relevant type of defect are micro-defects. These appear to take basically three forms: (a) small spots, (b) line defects, and (c) darker areas which appear within the central region of the specimen. The small spots are generally detected when their size is around $0.5 \mathrm{~mm}$ diameter, or bigger. The line defects in many cases lead to the development of small corrosion spots, and again their detectability appears to be size limited. The darker areas which appear within the central region of the specimen are suggested, from visual examination through the transparent epoxy layer, to be clusters of extremely small disbonds. As for the corrosion-driven edge-disbonds, the number and extent of the micro-defects increase with increasing time in water at $50^{\circ} \mathrm{C}$, as may be seen from Figures 2c and $2 \mathrm{~d}$.

Considering the ultrasonic scans in detail, after 27 days in water there are several relevant changes to be noted. The most important change is the disbonding initiating from the unsealed edges. (Outside of the bonded area, rapid corrosion of the free unseal ed edge can be seen, with large corrosion pits appearing. The marine paint that was used to seal two of the edges can al so be seen. This paint was applied after the initial scan but prior to exposure; but its thickness was not uniform and some corrosion occurred under it, which explains the mottled appearance on the scans.) However, closer observation shows that there are also micro-defects appearing under the epoxy in the form of some small dark spots. These are apparently quite randomly distributed, with no apparent difference being seen with proximity to sealed or unsealed, flush or recessed edges. (Unfortunately many of these small, micro-defects are more easily seen on the computer screen than the printed figure.) Some of these small spots have been highlighted on scans taken at longer exposure periods (e.g. Fig. 2c) but close scrutiny of the scan after 27 days shows initial signs of these micro-defects, i.e. relatively small defects which are isolated from adjacent defects and occur throughout the specimen. As the exposure time increases, the general trend is for many of the features seen after 27 days of exposure to increase in number and to grow in size. 
Fig. 3 shows the final scan taken on this specimen after 223 days in water, together with higher resolution scans of some of the micro-defects and a photograph looking through the epoxy. The high-resolution scans show that it is possible to detect micro-defects that are less than about 1 $\mathrm{mm}$ in diameter, with detail considerably below this being evident; i.e. dimensions of the order of $0.1 \mathrm{~mm}$, although this is smaller than could be reliably detected. It is important to note that, although the scan pitch is as small as $0.025 \mathrm{~mm}$ for the finest scan, the focal spot size of the probe remains unchanged for all of the normal incidence scans. Changing the scan pitch from 0.1 to 0.025 mm primarily helps by giving some spatial averaging, making smaller micro-defects easier to identify but no sharper. The photograph shows many more micro-defects than are apparent from the scans. Some of these are surface marks that are not seen on the ultrasonic image as it is focussed on reflections from the adhesive/adherend interface. However, many of the spots and lines seen in the photograph, particularly in the top left and bottom right hand corner regions, are at the interface, but are too small to be detected with the resolution of the ultrasonic transducer employed. Finally, from the results shown in Figs. 2 and 3, it is very noteworthy that there was no evidence of a gradual change in the normal-incidence reflection coefficient moving into an apparently well-bonded region from either the corrosion-driven edge-disbonds or the micro-defects.

Fig. 4 shows a comparison of a photograph of the failure surface from the two-layer grit-blast specimen (Fig. 4a) with the final normal-incidence scan (Fig. 4b). The specimen had been exposed for 223 days in water at $50^{\circ} \mathrm{C}$. The strips that were cut for the mechanical tests have been overlaid onto the ultrasonic image. The far right-hand strip was not tested mechanically as preparing the edge of the strip was considered to be too difficult due to the amount of disturbance to the epoxy layer because of the large edge-disbond present. Therefore, the original epoxy layer is still present on the photograph of this strip, see Fig. 4a. Considering the exposed failure surfaces shown in Fig. $4 \mathrm{a}$, it is interesting to note that many of the defects that were clearly visible on the ultrasonic scan are distinguishable on the failure surface, due to the presence of a very thin covering layer of retained epoxy. This suggests that there was a failure in the epoxy layer at, or very close to, the interface prior to the mechanical test; otherwise it would not have been detected on the scan focused on the interface region.

Fig. 5 shows a scanning electron micrograph of this specimen, together with a small section of the failure surface shown in the previous figure. This micrograph shows one of the lines that was visible in the photograph of the specimen taken at the time of the final ultrasonic scan shown in Fig. 
4a. The relatively rough surface generated by grit-blasting is evident, together with a line of epoxy covering the surface, see Fig. 5b. This suggests that the line defects seen in the photograph through the epoxy layer, and detected in the ultrasonic scans, might have been line-defects in the epoxy layer, close to the interface.

Correlating the above information to the toughness of the specimen, clearly the presence of corrosion-driven edge-disbonds resulted in a total loss of toughness in such degraded regions, but this type of defect was readily detected ultrasonically. However, if we ignore these regions, then the results shown in Table 1 reveal that the grit-blasted specimens lost some 54\% of their initial toughness, as measured using the DCB tests to determine the values of $G_{c}$ before and after exposure to water at $50^{\circ} \mathrm{C}$. Further, this loss of interphase toughness was found to occur relatively uniformly over the remainder of the specimen. For example, no significant differences occurred in the vicinity of a corrosion-driven edge-disbond, or indeed the micro-defects. This obviously correlates well with the observation that there was no evidence of a gradual change in the normal-incidence reflection coefficient upon moving into an apparently well-bonded region from either the edge-disbonds or the micro-defects. Thus, an important conclusion in the case of the two-layer grit-blast specimens, is that the detection of the isolated micro-defects was the only direct indicator that the interphase of the specimen had been weakened by the ingressing water molecules.

\subsection{Chromic-acid etched (CAE) specimens}

Fig. 6 shows two high-resolution scans from a two-layer CAE sample after 154 and 194 days in water at $50^{\circ} \mathrm{C}$, which illustrates that micro-defects remote from the edges are again apparent. These defects can readily be detected given a sufficiently high resolution scan. Fig. 6a shows a spot microdefect and, as with the grit-blast specimens, there were other types of micro-defects, particularly line-defects of the type shown in Fig. 6b. However, the number of micro-defects were very few in number, and this is reflected in the very good retention of toughness, $G_{c}$, of these specimens after water exposure at $50^{\circ} \mathrm{C}$ for 194 days, as may be seen in Table 1.

\subsection{Phosphoric-acid anodised (PAA) specimens}

Fig. 7 shows the mechanical test results, together with photographs of the failure surfaces, for the 'wet' and 'dry' two-layer PAA specimens. Figs. 7a and b show the maps of the fracture toughness; where the value of $G_{c}$ is shown as a function of crack length for the $10 \mathrm{~mm}$ wide fracture specimens which were cut from the two-layer PAA specimens. Thus, Figs. 7a and b give information on measured values of $G_{c}$ which are assigned to spatially discrete positions within the specimen. The 
missing information on these maps is where (a) corrosion-driven edge-disbonds had occurred prior to the specimen being cut-up for the fracture tests, which of course resulted in that part of the specimen strip having zero toughness, or (b) unstable, stick-slip crack growth had occurred during the fracture tests, so that no value of $G_{c}$ could be determined for this fracture test strip, or portion of the strip.

The average fracture toughness for the 'dry' specimen was $55 \mathrm{~J} / \mathrm{m}^{2}$, with the value of $\mathrm{G}_{\mathrm{c}}$ being relatively uniform across the specimen, as would be expected. For the 'wet' specimen, which had been exposed to water at $50^{\circ} \mathrm{C}$ for 393 days, the average toughness was reduced to $30 \mathrm{~J} / \mathrm{m}^{2}$ after 393 days in water at $50^{\circ} \mathrm{C}$. As may be seen from Fig. 7a, for the 'wet' fracture specimens the loss of toughness is also relatively uniform across the specimen. Indeed, there was no significant extra loss of toughness in a region adjacent to a corrosion-driven edge-disbond or a micro-defect.

As indicated in Figs. 7c and d, sections of the failure surfaces were analysed using X-ray photoelectron spectroscopy (XPS). These analyses revealed that failure in the 'dry' tests was mainly through the epoxy adhesive, albeit close to the interface. However, after environmental attack, the locus of failure of the two-layer specimens moved closer to the adhesive/adherend interface, with failure also occurring either at the interface or in the oxide layer.

The ultrasonic scans again revealed there was no evidence of a gradual change in the normalincidence reflection coefficient moving into an apparently well-bonded region from either the edgedisbonds or the micro-defects. This conclusion obviously correlates to the results shown in Fig. 7a, where there was no significant extra loss of toughness in any region adjacent to a corrosion-driven edge-disbond or a micro-defect. Further, the detection of micro-defects via the ultrasonic scans correlates in a semi-quantitative manner with the retained toughness of $55 \%$ which was recorded for the PAA two-layer specimens; i.e. the number of micro-defects detected is intermediate in number between that for the grit-blast and the CAE specimens, as is the average toughness which is retained.

Finally, the relatively poor performance of the PAA joints is at first sight somewhat surprising, but this has been shown to arise from these joints being prepared without the primer which is typically used in the aerospace standard procedure [7]. The primer was omitted in order to reduce the number of layers in the joint and so to simplify the ultrasonic signals in these tests. 


\subsection{Chromic-acid anodised (CAA) specimens}

Fig. 8 shows the progression of degradation for a two-layer CAA specimen. There is a clear progression of a corrosion-driven edge-disbond initiating on the unsealed flush edge, adjacent to the unsealed recessed edge. This is accompanied, after about 100 days of exposure to the aqueous environment, by a second edge-disbond which develops from the unsealed recessed edge. However, the CAA specimen differed from the other surface pretreated two-layer specimens in that at no point was there any visual or ultrasonic evidence of any micro-defects during the 465 days that this specimen was in water, which is far longer than any of the other two-layer specimens. This is in excellent correlation with the observation that there is no loss of toughness in the CAA two-layer specimens, see Table 1.

The higher resolution scan shown in Fig. 8d after 103 days of exposure shows a detailed view of the corrosion-driven edge-disbond. This demonstrates that there is a 'sharp edge' between the edge-disbond and what appears to be undamaged epoxy and epoxy/adherend interfacial regions. Indeed, this suggestion that there is a 'sharp edge' at the leading front of the edge-disbond is again supported by there being no significant extra loss of toughness in a region adjacent to this corrosion-driven edge-disbond. (However, as seen on other specimens, gross corrosion on the now debonded aluminium-alloy surface associated with the edge-disbond region does lead to a reduction in ultrasonic signal amplitude.) It is interesting to note that, although no primer was used with the CAA pretreatment, excellent durability of the two-layer specimen was recorded, unlike the case for the unprimed PAA pretreatment. It is suggested that this difference arises from the different morphologies of these oxide layers [1-7]. The CAA generated oxide is far thicker and possesses far smaller pores which do not pass completely through the oxide layer compared to the oxide morphology generated by the PAA pretreatment. Indeed, a CAA pretreated oxide is known [1] to not always require a primer in order to impart a good durability to an adhesively-bonded structure.

\section{RESULTS: THREE-LAYER SPECIMENS}

\subsection{Ultrasonic Scans}

The results from the three-layer specimens are summarised in Table 2. In these specimens the extent of corrosion-driven edge-disbonding was much lower than that seen in the corresponding two-layer specimens, as may be readily observed from comparing the results shown in Tables 1 and 2 . This is undoubtedly due to the addition of the second adherend in the three-layer specimens increasing the stiffness of the system. This prevents the epoxy layer from being forced away from the aluminium 
surface by the growth of corrosion products on the aluminium surface. These voluminous corrosion products act to lever the joint open in the two-layer specimen and this accelerates the rate of edge disbonding. The grit-blast specimen was exposed for a shorter time period than the other specimens because relatively large edge disbonds became apparent after a relatively short exposure time, whereas there was minimal evidence of edge-disbonds on the PAA specimen even after a very long exposure time.

The normal-incidence ultrasonic technique did not detect any micro-defects, or any other changes, remote from the edges of any of the three-layer specimens, although the scans did readily detect disbonds growing in from the edges of the grit-blast and CAE three-layer specimens, as discussed above. Thus, a main conclusion to be drawn is that the micro-defects, which were indicative of environmental attack on the interphase regions and of an accompanying decrease in interface toughness for the two-layer specimens, were not detected via the ultrasonics scans in the grit-blast and PAA three-layer specimens, nor indeed in any of the three-layer specimens.

Nevertheless, the fracture surfaces of the failed mechanical tests of the grit-blast and PAA specimens showed fine line-defects of epoxy; i.e. showed signs of micro-defects. These surface features, as shown in Fig. 9d for example, were similar to those which corresponded to the microdefects which were detectable by the ultrasound techniques in the two-layer specimens; although they were smaller in number and in size on the failure surfaces of the grit-blast and PAA three-layer specimens. Therefore, it is possible that micro-defects remote from the edges were present in the three-layer specimens that failed interfacially and suffered a decrease in interphase toughness, but clearly they were not able to be detected ultrasonically. This possibility is further supported from the visual observations on the two-layer specimens, which clearly revealed that many more microdefects were present than were detected ultrasonically - only the wider lines or larger spots being visible with ultrasound. (The two opaque adherends of the three-layer specimens meant, of course, that visual inspection of the interface was not possible with the three-layer specimens. Hence, for the three-layer specimens, only the appearance of the failure surfaces of the specimens which fractured interfacially can assist on this point.)

Thus, it would seem that micro-defects were present in the grit-blast and PAA three-layer specimens. There are two possible reasons for the apparently smaller number and size of microdefects in the grit-blast and PAA three-layer specimens, compared to the corresponding two-layer specimens where a loss of toughness, $G_{c}$, was also observed upon exposure of the specimens to 
water at $50^{\circ} \mathrm{C}$. Firstly, it is possible that the extra stiffness of the three-layer samples restricted the growth of the micro-defects to below that required for ultrasonic detection. Secondly, since the concentration of water in the adhesive layer at any time would be far lower in the three-layer specimens than the two-layer specimens (see below), it may be that this factor limited the initiation and subsequent growth of the micro-defects in the three-layer specimens to below that required for ultrasonic detection.

\subsection{Mechanical Tests}

The mechanical tests on the unexposed, 'control', specimens showed that they all failed via cohesive fracture through the adhesive layer. From a visual assessment, the three-layer grit-blast and PAA specimens exposed to water failed interfacially, whilst the failure of the CAE and CAA specimens remained cohesive through the adhesive layer. (Again, the calculation of the average toughness, $G_{c}$, for the degraded samples excluded the regions of any edge-disbonding.) However, as may be seen from the results shown in Table 2, the exposed specimens that failed cohesively still experienced some loss of toughness. This complex behaviour was found to be due to the deleterious effects of heat on the properties of the epoxy adhesive layer, but without the benefits from the adhesive layer being able to readily absorb moisture and so become plasticised, which would be accompanied by an increase in toughness, as seen in the two-layer specimens. This was verified by exposing specimens to $50^{\circ} \mathrm{C}$ but under low humidity (i.e. 'dry') conditions [30]. (It should be noted that during these experiments no edge-disbonding or micro-defects of any type were observed.)

However, whilst a full quantitative picture is difficult to discern, clearly the three-layer gritblast and PAA surface pretreatments give joints where environmental attack has occurred in the interphase regions. This may be seen in Table 2 by the change from a cohesive to a visually interfacial locus of failure after water exposure, which is accompanied by a significant decrease in the toughness, $G_{c}$, of the joint. As an example, the mechanical test data are shown in Fig. 9 for the three-layer PAA specimen. As may be seen, there are some isolated regions where cohesive failure through the adhesive layer is observed and these regions are associated with a relatively high value of $G_{c}$. For the remainder of the joint, failure in the interphase regions of the joint occurred and the value of $G_{c}$ was relatively lower but uniform in value, see Fig. 9a. Since, the failure of the 'dry' three-layer PAA joint was always via cohesive failure through the adhesive layer, then clearly the presence of the aqueous environment has led to change in the locus of failure and associated lower value of $G_{c}$. Fig. 9d illustrates the lines of epoxy adhesives which were retained on the aluminium- 
alloy adherend. These are very similar in appearance, both visually and via scanning electron microscopy, to those found on the two-layer specimens, see Fig. 5.

Again, the relatively poor performance of the PAA joints is at first sight somewhat surprising, but this has been shown to arise from these joints being prepared without the primer which is typically used in the aerospace standard procedure [7]. As commented above, the primer was omitted in order to reduce the number of layers in the joint and so to simplify the ultrasonic signals in these tests. Also, again as was discussed above, it is interesting to note that, although no primer was used with the CAA pretreatment, durability of the three-layer specimen was recorded, unlike the case for the unprimed PAA pretreatment.

\section{DISCUSSION}

\subsection{Two-layer Specimens: Summary}

In the two-layer specimens, two main types of defects were detected ultrasonically in the aged specimens: corrosion-driven edge-disbonds and micro-defects. Edge-disbonds were simple to detect using the normal-incidence ultrasonic technique. Micro-defects were seen in regions remote from the edges and these small-scale, isolated defects appeared to take basically three forms. Firstly, there were small spots which were generally detected ultrasonically when their size was around 0.5 $\mathrm{mm}$ diameter or bigger. Secondly, there were line defects, which in many cases led to the development of small corrosion spots. Again their detectability appeared to be size limited. Thirdly, there were also darker areas which appeared within the central region of the specimen, which visual examination through the transparent epoxy suggested were clusters of extremely small disbonds. Many of these micro-defects could be seen visually through the transparent epoxy adhesive which coated the two-layer specimen and some, but not all, could be detected in the normal-incidence ultrasonic scans.

Correlating the above information to the toughness of the two-layer specimens, clearly the presence of corrosion-driven edge-disbonds resulted in a total loss of toughness in such degraded regions, but this type of defect was readily detected ultrasonically. However, if we ignore these regions, then the results shown in Table 1 reveal that the two-layer grit-blasted and PAA specimens lost a significant amount of their initial toughness, and this loss of interphase toughness was found to occur relatively uniformly over the remainder of the specimens. The detection of isolated microdefects was the only indicator that the interphase of the specimen had been attacked, and so weakened, by the ingressing water molecules. Indeed, the number of, and area occupied by, such 
defects could be correlated semi-quantitatively to the loss of interphase toughness, $G_{c}$. It is very noteworthy, that there was no evidence of a gradual change in the normal-incidence reflection coefficient moving into an apparently well-bonded region from either the edge disbonds or the micro-defects. This observation correlates well with the $G_{c}$ measurements via the fracture toughness maps, which indicated that there was no extra extent of weakening of the interphase regions adjacent to either of these types of defects.

\subsection{Three-layer Specimens: Summary}

In the case of the three-layer specimens, the extent of corrosion-driven edge-disbonding was much lower than that seen in the corresponding two-layer specimens. Another major finding was that no micro-defects were detected ultrasonically in any of the three-layer specimens, although examination of the failure surfaces from the mechanical tests of the grit-blasted and PAA threelayer specimens, that failed interfacially and suffered a decrease in the interphase toughness, $G_{c}$, suggested that some micro-defects may well have been present. However, if this was the case, then clearly they were too small to be detected at the spatial resolution of the ultrasonic scans. (It would be difficult to improve the spatial resolution by increasing the frequency of the normal-incidence inspection because the attenuation in the adherend would increase rapidly.) Thus, unlike the twolayer specimens, for the three-layer specimens the occurrence of environmental attack and the associated loss of toughness, $G_{c}$, could not be anticipated by the detection of micro-defects via ultrasonic inspection.

Finally, again, there was no evidence of a gradual change in the normal-incidence reflection coefficient moving into an apparently well-bonded region from the edge disbonds, when present. This observation correlates with the $G_{c}$ measurements via the fracture toughness maps, which indicated that there was no extra extent of weakening of the interphase regions adjacent the corrosion-driven edge-disbonds. However, the ultrasonic scans have clearly failed to detect any changes at all in the interphase regions which would indicate the general loss of interphase toughness seen with the three-layer grit-blast and PAA specimens.

\subsection{Mechanisms of Failure}

\subsubsection{Introduction}

The relative performance of the different pretreatments used in the present work followed that expected from the literature [e.g. 1, 2]: the CAA pretreatment giving the most durable two- and three-layer specimens and the grit-blasting pretreatment giving the least durable. However, as noted 
above, the PAA treatment resulted in specimens which performed less well than expected and this has been shown to arise from these joints being prepared without the primer which is typically used in the aerospace standard procedure [7].

\subsubsection{Edge-disbonding}

The design of the specimens was deliberately undertaken such that some areas of the specimen would see the ingress of water faster than others. This was achieved through the use of a marine epoxy-based paint along two of the edges to seal them against the ingress of water. The variation in edge conditions of the joint could then be investigated and their significance determined.

Indeed, there were significant differences in the rate of degradation via edge-disbonding experienced by the different edges of the specimens. The edge-disbonding mechanism usually started at the unsealed flush edge, and was initiated by a region of corrosion which developed on the edge of the specimen and which undercut the epoxy layer. Growth of the corrosion products, which subsequently formed, forced the interface open to allow water ready entry to these regions. The specimens with different pre-treatments showed edge-disbonding to a greater or lesser extent dependent on the corrosion protection offered by the pretreatment employed, in accordance with published studies as commented above. Edge-disbonds were easily detected ultrasonically. In the case of the two-layer specimens the clear epoxy layer also allowed a visual inspection to be made. Such visual assessments confirmed that the extent of disbonding detected ultrasonically was a very accurate measure of the development of this failure mechanism. Ultrasonic scans from the two-layer grit-blast and CAA specimens, which represent the least and most durable specimens, are shown in Figs. 2 and 8.

One major difference between the two- and three-layer-specimens was that in the case of the three-layer specimens the extent of such edge-disbonding was much lower than that in the corresponding two-layer specimens. This was undoubtedly due to the addition of the second adherend in the three-layer specimens increasing the stiffness of the system. This prevented the epoxy layer from being forced away from the aluminium surface by the growth of corrosion products on the aluminium surface. This excessive, voluminous growth tended to lever the joint open, accelerating the rate of edge-disbonding.

However, whilst clearly this edge-disbonded region of the specimen had zero toughness, the presence of edge-disbonding did not necessarily indicate that the remaining interphase regions of 
the specimens had suffered any environmental attack and weakening by the ingressing moisture; see, for example, the results in Table 1 for the two-layer CAA specimens. Conversely, the absence of the corrosion-driven edge-disbonding mechanism did not necessarily mean that the remaining regions of the joint were free from environmental attack and weakening; see, for example, the threelayer PAA joint, Table 2.

\subsubsection{Micro-defects}

The micro-defects detected in all the two-layer specimens appeared to take basically three forms, as noted above in detail. Firstly, there were small spots which were generally detected when their size was around $0.5 \mathrm{~mm}$ diameter, or bigger. Secondly, there were line-defects, which in many cases led to the development of small corrosion spots. Again their detectability appeared to be size limited. Thirdly, there were also darker areas which appeared within the central region of the specimen, which visual examination through the transparent epoxy suggested were clusters of extremely small disbonds. For the two-layer specimens, the detection of micro-defects in the specimens via the ultrasonic scans could be correlated in a semi-quantitative manner with the extent of environmental attack upon the interphase regions of the specimens and the associated value of $G_{c}$.

The micro-defects were clearly present in a greater number and to a greater extent in the twolayer specimens than the corresponding three-layer specimens. Indeed, no micro-defects were ever detected in the ultrasonic scans from the three-layer specimens, although, as noted above, they were thought to be present in at least some of the three-layer specimens. This difference between the twoand three-layer specimens was most likely due to the far more rapid ingress of water to the interphase regions for the 'open-faced' two-layer specimens, compared to that for the three-layer specimens. This is shown in detail in Fig. 10, as discussed below. However, again, the addition of the second adherend in the three-layer specimens increasing the stiffness of the system might have affected the formation of such micro-defects. For example, increasing the stiffness would prevent the formation of micro-voids at, or close to, the interface.

However, whatever the reason, the number and extent of micro-defects in the three-layer specimens, coupled with the greater difficulty of detecting them, compared to the two-layer specimens led to these micro-defects being undetectable via the ultrasonic scans for the three-layer joint specimens. Thus, for the three-layer PAA joints, the ultrasonics could not detect any feature at all which would indicate that these joints had indeed suffered significant weakening of their interphase regions, and an associated loss of interphase toughness, $G_{c}$, upon exposure to the 
aqueous environment, whilst, for the three-layer grit-blast specimen, the ultrasonic scans could only detect corrosion-driven edge-disbonds, which are not indicative of a loss of interphase toughness, as may be seen from Tables 1 and 2 .

Finally, the formation of micro-defects in the form of debonds widely dispersed across a specimen, which develop during environmental degradation and are associated with a loss of toughness, has also been reported by Moidu et al. [31,32]. Lavrentyev and Rokhlin [33] have also suggested that environmental degradation occurs via the formation of a large number of microdefects widely dispersed across the specimen, rather than a single, steadily advancing defect.

\subsubsection{Loss of Interphase I ntegrity}

It is very noteworthy that there was no evidence of a gradual change in the normal-incidence reflection coefficient moving into an apparently well-bonded region from either the edge disbonds or the micro-defects. This observation at first sight agrees with the $G_{c}$ measurements via the fracture toughness maps. These maps indicated that for both two- and three-layer specimens there was no extra extent of weakening of the interphase regions adjacent to either of these types of defects; i.e. the remaining bonded regions after water exposure revealed a relatively uniform decrease in the value of $\mathrm{G}_{c}$; see for example Figs. 7 and 9. However, the ultrasonic scans have clearly failed to detect any changes at all in the interphase regions which would indicate that this general loss of toughness seen with some of the specimens had occurred, apart from the detection of isolated micro-defects in the case of the two-layer specimens.

\subsubsection{Rate of Water Ingress}

It is known that epoxy adhesives absorb water [34], and it has been found that water diffusion into the epoxy layer may govern the rate of joint degradation [35]. Therefore, water uptake experiments were conducted on a sheet of the cured epoxy material which gave a water diffusion coefficient at $50^{\circ} \mathrm{C}$ of $2.04 \times 10^{-13} \mathrm{~m}^{2} \mathrm{~s}^{-1}$ [36]. This was then used to predict water concentrations in both the twoand three-layer specimens as a function of time and distance into the specimen, assuming Fickian diffusion [37].

The predictions for water ingress into the three-layer specimen are shown in Fig. 10a where the fractional water content after 700 days exposure to water at $50^{\circ} \mathrm{C}$ is plotted as a function of the distance into the joint from the edge of the specimen. (In Fig. 10a the term $\mathrm{C}$ represents the water concentration in the adhesive layer of the three-layer specimen as a function of distance into the 
specimen after 700 days in water at $50^{\circ} \mathrm{C}$ and the term $\mathrm{C}_{1}$ is the water concentration at complete saturation in the epoxy material, which was $2.5 \mathrm{w} / \mathrm{w} \%$ [36].) The fact that water is only predicted to reach a measurable concentration within the outer one-third of the regions of the joint is very noteworthy, especially bearing in mind the relatively long exposure time used for these calculations compared to the shorter total times for which the three-layer specimens were actually exposed to water, see Table 2.

On the other hand, Fig.10b reveals that for the open-faced epoxy, two-layer, specimens the water would diffuse through the epoxy layer and reach the epoxy/aluminium-oxide interface at its equilibrium, saturation concentration (for the bulk epoxy) after only about 350 days of exposure to water at $50^{\circ} \mathrm{C}$. (In Fig. $10 \mathrm{~b}$ the term $\mathrm{C}_{0}$ represents the water concentration in the two-layer specimen at the epoxy/aluminium-alloy interface as a function of time.) Thus, it should be noted that, for the two-layer PAA and CAA specimens the concentration of water would be uniform across the specimen prior to the total number of days of exposure to water at $50^{\circ} \mathrm{C}$ having been reached, see Table 1.

From the above discussions, several factors indicate that water diffusion through the bulk epoxy was not the primary rate-determining step in the degradation of at least some of the present specimens. For example, firstly, it was observed that most regions of the two-layer CAA specimen exposed to water showed an increase in toughness compared with the 'dry' specimen, despite it being immersed in water for nearly 500 days, see Table 1. Now, Fig. 10b reveals that this time period was more than sufficient to saturate completely the epoxy layer immediately adjacent to the interface. However, it is well established $[35,38]$ that a given, critical, concentration of water is the not the only requirement for environmental attack upon an interphase. Clearly, the nature of the interphase is of prime importance, especially (a) the nature of the molecular bonding across the epoxy/aluminium-oxide interface and (b) the inherent stability and corrosion resistance of the aluminium-alloy oxide layer. Both of these factors are greatly influenced by the surface pretreatment employed. Thus, clearly the present work supports the general observation that a CAA pretreatment may impart an excellent durability to an epoxy/aluminium-alloy interface.

Secondly, on the other hand, the interfacial failure and associated loss of toughness of both the three-layer grit-blast and PAA specimens across their entire area (see Table 2 and Fig. 9) cannot be readily explained by water diffusion through the (bulk) adhesive layer, since the water concentration in the central regions of these joints via such a route would be virtually zero after the total exposure 
times involved, i.e. compare the data shown in Table 2 and Fig. 10a. These results therefore suggest that such water diffusion through the (bulk) epoxy adhesive layer is too slow to explain the interfacial failure of these three-layer specimens and imply that water is reaching the central regions of the three-layer joints via a faster route. Two possibilities are suggested.

One possibility is the formation of micro-cracks, and other micro-defects, in the epoxy adhesive due to the action of heat and moisture absorption, as has been discussed by Comyn [34]. He cites several examples of cases where water may exist in micro-cavities in the adhesive layer, these being in the form of either cracks or voids. It is therefore possible that the micro-defects which developed in the specimens upon exposure to water are responsible for (a) the relatively rapid water transport in these specimens and (b) the water then degrading the joint in a relatively uniform manner, so accounting for the relatively uniform toughness loss seen in some of the threelayer specimens. It is also possible that these micro-defects are formed to release either swelling or contraction stresses that have developed in the adhesive layer of joints. The fact that the microdefects have appeared in some specimens after so little time in water (see, for example, Fig. 2) suggests that they are more likely to be caused by the effects of heat than from swelling solely due to water uptake. However, as commented earlier, they have only been seen on specimens exposed to water and not in specimens that have been kept in 'dry' heat [30], suggesting that the absorption of water by the adhesive layer is a necessary requirement for their appearance. (Their appearance may also be related to the very brittle, simple epoxy used in these tests; it would be interesting to investigate whether they are also seen with tougher adhesives. It is also necessary to explain why the occurrence of these flaws in the epoxy layer, several micrometers from the interface, appears to be a function of the adherend pretreatment.) The second possibility is that in some adhesive systems the diffusion of water along the adhesive/adherend interface is far quicker than that through the bulk adhesive layer [39-41]. Indeed, using an elegant Fourier-transform infrared multiple internalreflection technique, Linossier et al. [41] not only demonstrated this to be possible, but they also observed that the rate of interfacial diffusion of water was a function of the surface pretreatment used for the adherend prior to bonding.

\section{CONCLUSIONS}

Normal-incidence ultrasonic scans were conducted on two- and three-layer adhesive-joint specimens exposed to water at $50^{\circ} \mathrm{C}$ for periods of up to 18 months. The joints consisted of aluminium-alloy adherends which were subjected to one of four different surface pretreatments prior to being coated (for the two-layer specimens) or bonded (for the three-layer specimens) with 
an epoxy polymer. Techniques were developed to measure the fracture toughness, $G_{c}$, of the specimens before and after water exposure; so that fracture toughness maps could be ascertained, where the value of $G_{c}$ could be assigned approximately to spatially discrete positions within the specimen. The relative performance of the different pretreatments used in the present work followed that expected from the literature: the chromic-acid anodised (CAA) surface pretreatment giving the most durable two- and three-layer specimens and the grit-blasting pretreatment giving the least durable.

In the two-layer specimens, two main types of defects were detected: corrosion-driven edgedisbonds and micro-defects. The edge-disbonding mechanism usually started at the unseal ed flush edge, and was initiated by a region of corrosion which developed on the edge of the specimen and which undercut the epoxy layer. Growth of the corrosion products, which subsequently formed, forced the interface open to allow water ready access to these regions. Edge-disbonds caused a total loss of interphase integrity but were easily and accurately detected ultrasonically. Micro-defects were detected in regions remote from the edges and these small-scale, isolated defects took several forms. In the case of the two-layer specimens, the detection of these isolated micro-defects was the only indicator that the interphase of the specimen had been attacked and weakened by the ingressing water molecules. Indeed, the number of such defects correlated in a semi-quantitative manner to the loss of interphase toughness.

In the case of the three-layer specimens, the extent of edge-disbonding was much lower than that seen in the corresponding two-layer specimens. This was undoubtedly due to the addition of the second adherend in the three-layer specimens increasing the stiffness of the system. This prevented the epoxy layer from being forced away from the aluminium surface by the voluminous growth of corrosion products on the aluminium surface. This growth tended to lever the joint open in the twolayer specimen and so accelerated the rate of edge-disbonding. Also, no micro-defects were detected ultrasonically in any of the three-layer specimens. However, examination of the failure surfaces from some of the three-layer specimens suggested that micro-defects may have been present but that they were too small to be detected at the spatial resolution of the ultrasonic scans. Therefore, for the three-layer joint specimens, there were no indicators from the ultrasonic scans which could reveal whether the joint had suffered attack and weakening of the interphase regions by the ingressing moisture. 
Thus, to summarise, the ultrasonic scans could readily detect any corrosion-driven edgedisbonds present in the either the two- or threelayer specimens, and such defects resulted in a complete loss of interphase integrity. However, whilst the ultrasonic scans did detect the presence of micro-defects in the two-layer specimens, which appeared to correlate semi-quantitatively with the extent of toughness loss upon water exposure, the scans clearly failed to detect any changes in the interphase regions which would indicate the general loss of toughness seen with the three-layer grit-blast and PAA specimens. Finally, for all the tests conducted it was very noteworthy that there was no evidence of a gradual change in the normal-incidence reflection coefficient when moving into an apparently well-bonded region from either the edge disbonds or the micro-defects, when present. This observation correlated with the $\mathrm{G}_{\mathrm{c}}$ measurements via the fracture toughness maps, which indicated that there was no extra extent of weakening of the interphase regions adjacent to the corrosion-driven edge-disbonds or micro-defects.

\section{Acknowledgements}

The authors would like to thank Professor J.F. Watts (University of Surrey) for assistance with the XPS studies. The authors are grateful for support of the work by DERA (UK). 


\section{References}

1. Kinloch, A.J. (Ed.), Durability of Structural Adhesives (Applied Science, London and New York, 1983).

2. Davies, R.J. and Kinloch, A.J., in Adhesion 13, Allen, K.W., Ed. (Elsevier, London, 1989) p.8.

3. O'Sullivan, J.P. and Wood, G.C., Proc. Roy. Soc. Lond. A317, 511(1970).

4. Thompson, G.E., Furneaux, R.C., Goode, J.S. and Wood, G.C., Trans. Inst. Metal Finishing 56,159 (1978).

5. Thompson, G.E. and Wood, G.C. (1983) in Corrosion: Aqueous Processes and Passive Films, Scully, J.C., Ed. (Academic Press, London, 1983).

6. Xu, Y., Thompson, G.E. and Wood, G.C., Trans. Inst. Metal Finishing 63, 98 (1985).

7. Kinloch, A.J., Little, M.S.G. and Watts, J.F., Acta Materilia 48, 4543 (2000).

8. Thompson, R.B. and Thompson, D.O., J. Adhesion Sci. Technol. 5, 583 (1991).

9. Cawley, P., Pialucha, T.P. and Lowe, M.J.S. in Review of Progress in Quantitative NDE, Vol. 12, Thompson, D.O and Chimenti, D.E., Eds. (Plenum Press, New York, 1993) p.1531.

10. Rokhlin, S.I. and Marom, D., J. Acoust. Soc. Amer. 80, 585 (1986).

11. Pilarski, A. and Rose, J.L., NDT International 21, 241 (1988).

12. Jiao, D. and Rose, J.L., J. Adhesion Sci. Technol. 5, 631 (1991).

13. Cawley, P., Pialucha, T.P. and Zeller, B.D., Proc. Royal Soc. Lond. A452, 1903 (1996).

14. Rokhlin, S.I. and Wang, Y.J., J. Acoust. Soc. Amer. 89, 503 (1991).

15. Bar-Cohen, Y. and Mal, A.K., in Review of Progress in Quantitative NDE, Vol. 9B, Thompson, D.O and Chimenti, D.E., Eds., (Plenum Press, New York, 1990) p.1271.

16. Jungman, A., Guy, P., Nayfeh, A. and Quentin, G., in Review of Progress in Quantitative NDE, Vol. 10B, Thompson, D.O and Chimenti, D.E., Eds., (Plenum Press, New York, 1991) p.1319.

17. Claus, R.O. and Kline, R.A., J. Appl. Phys. 50, 8066 (1979).

18. Rokhlin, S.I., Hefets, M. and Rosen, M., J. Appl. Phys. 52, 2847 (1981).

19. Pilarski, A., Materials Evaluation 43, 765 (1985).

20. P.B. Nagy and L. Adler, in Review of Progress in Quantitative NDE, Vol. 10B, Thompson, D.O and Chimenti, D.E., Eds. (Plenum Press, New York, 1991) p.1295. 
21. Pilarski, A., Rose, J.L. and Balasubramanian, K., J. Acoust. Soc. Amer. 87, 532 (1990).

22. Wang, W. and Rokhlin, S.I., J. Adhesion Sci. Technol. 5, 647 (1991).

23. B. Li, M. Hefetz and S.I. Rokhlin, in Review of Progress in Quantitative NDE, Vol. 11B, Thompson, D.O and Chimenti, D.E., Eds. (Plenum Press, New York, 1992) p.1221.

24. Lavrentyev, A.I. and Rokhlin, S.I, in Review of Progress in Quantitative NDE, Vol. 13, Thompson, D.O and Chimenti, D.E., Eds. (Plenum Press, New York, 1994) p.1539.

25. Jackson, R.J., Kinloch, A.J., Gardham, L.M. and Bowditch, M.R., in Proc. $19^{\text {th }}$ Annual Meeting of the Adhesion Soc., Ward T., Ed. (Adhesion Soc., USA, 1996) p.147.

26. Wylde, J.W. and Spelt, J.K., Int. J. Adhesion and Adhesives 18, 237 (1998).

27. Cawley, P., Kinloch, A.J. and Vine, K., submitted for publication (2001).

28. Blackman, B.R.K., Dear, J.P, Kinloch, A.J. and Osiyemi, S., J. Materials Science Letters 10, 253 (1991).

29. Duer, R., Katevatis, D., Kinloch, A.J. and Williams, J.G., Int. J. Fracture 75, 157 (1995).

30. Vine, K. The Non-destructive Testing of Adhesive Joints for Environmental Degradation, PhD Thesis (Imperial College, University of London, 1999).

31. Moidu, A.K., Sinclair, A.N. and Spelt, J.K., J. Adhesion 65, 239 (1998).

32. Moidu, A.K., Sinclair, A.N. and Spelt, J.K., Research in Non-destructive Evaluation, 11, 81 (1999).

33. Lavrentyev, A.I. and Rokhlin, S.I., J. Applied Physics 76, 4643 (1994).

34. Comyn, J., in Durability of Structural Adhesives, Kinloch, A.J., Ed. (Applied Science, London and New York, 1983) p.85.

35. Gledhill, R.A., Kinloch, A.J., and Shaw, S.J., J. Adhesion 11, 3 (1980).

36. Pialucha, T.P., Imperial College, University of London, Personal communication (1992).

37. Crank, J., The Mathematics of Diffusion (Oxford University Press, 2nd ed, 1975).

38. Kinloch, A.J., Adhesion and Adhesives: Science and Technology (Chapman and Hall, London, 1987).

39. Nguyen, T., Byrd, E., Bentz, D. and Lin, C., Progress in Organic Coatings 27, 181 (1996).

40. Zanni-Deffarges, M.P. and Shanahan, M.E.R., Int. J. Adhesion Adhesives 15, 137 (1995).

41. Linossier, I., Gaillard, F., Romand, M. and Nguyen, T., J. Adhesion 70, 221 (1999). 
Figure Captions

Figure 1. Two and three-layer specimen design.

Figure 2 Normal-incidence scans from two-layer grit-blast specimens after exposure for: (a) 0 days (b) 27 days (c) 67 days (d) 117 days exposed to water at $50^{\circ} \mathrm{C}$.

Figure 3 Two-layer grit-blast specimen after 223 days exposed to water at $50^{\circ} \mathrm{C}$ : (a) photograph; (b) ultrasonic scan at $0.25 \mathrm{~mm}$ scan pitch; (c) ultrasonic scan at $0.1 \mathrm{~mm}$ scan pitch; (d) ultrasonic scan at $0.025 \mathrm{~mm}$ scan pitch.

Figure 4 Two-layer grit-blast specimen after 223 days in water: (a) failure surface; (b) normalincidence ultrasonic scan.

Figure 5 Failure surface from two-layer grit-blast specimen: (a) photograph; (b) scanning electron micrograph.

Figure 6 Normal-incidence ultrasonic high-resolution scans of (a) a small spot micro-defect taken from a CAE specimen after 154 days in water at $50^{\circ} \mathrm{C}$ and (b) a narrow line micro-defect from the same CAE specimen after 194 days in water at $50^{\circ} \mathrm{C}$.

Figure $7 \quad$ Mechanical test results from the two-layer PAA specimens. Fracture toughness map from: (a) specimen after 393 days in water at $50^{\circ} \mathrm{C}$; (b) control 'dry' specimen. (c) photograph of the failure surface corresponding to (a); (d) photograph of the failure surface corresponding to (b). (The bottom and right-hand edges were the unsealed edges.)

Figure 8 Normal-incidence ultrasonic scans from the two-layer CAA specimen after: (a) 0; (b) 103; (c) 175; (e) 309; (f) 465 days in water at $50^{\circ} \mathrm{C}$; (d) high resolution scan of an edge-disbond in (b).

Figure 9 Mechanical test results from three-layer PAA specimens. Fracture toughness map from: (a) specimen after 566 days in water; (b) control specimen. (c) failure surface corresponding to (a); (d) expanded view of section from (c) showing lines of epoxy on failure surface.

Figure 10 Predicted water concentration in the specimens as a fraction of saturation for: (a) in the epoxy layer of a three-layer specimen as a function of spatial position after 700 days in water at $50^{\circ} \mathrm{C}$; and (b) at the aluminum/epoxy interface of a two-layer specimen as a function of time in water at $50^{\circ} \mathrm{C}$. 
Table 1. Summary of results from two-layer specimens.

\begin{tabular}{|c|c|c|c|c|c|c|}
\hline \multirow[t]{2}{*}{ Specimen } & \multirow{2}{*}{$\begin{array}{c}\text { Total } \\
\text { exposure } \\
\text { time (days) }\end{array}$} & \multirow{2}{*}{$\begin{array}{c}\text { Edge-disbond } \\
\text { area }\left(\mathrm{mm}^{2}\right)\end{array}$} & \multirow{2}{*}{$\begin{array}{l}\text { Edge-disbond } \\
\text { rate }\left(\mathrm{mm}^{2} / \text { day }\right)\end{array}$} & \multicolumn{2}{|c|}{ Micro-defects } & \multirow{2}{*}{$\begin{array}{c}\text { Average } \\
\text { toughness } \\
\text { retained (\%) }\end{array}$} \\
\hline & & & & Number & $\begin{array}{c}\text { Total area } \\
\left(\mathrm{mm}^{2}\right)\end{array}$ & \\
\hline Grit blast & 223 & 1464 & 6.6 & 648 & 103 & 46 \\
\hline CAE & 185 & 685 & 3.7 & 20 & 4 & 96 \\
\hline PAA & 393 & 1274 & 3.2 & 176 & 16 & 55 \\
\hline CAA & 465 & 1013 & 2.2 & 0 & 0 & 126 \\
\hline
\end{tabular}

Table 2. Summary of results from the three-layer specimens.

\begin{tabular}{|c|c|c|c|c|c|c|}
\hline Specimen & $\begin{array}{c}\text { Total exposure } \\
\text { time (days) }\end{array}$ & $\begin{array}{c}\text { Edge-disbond } \\
\text { area } \mathbf{( m m}^{\mathbf{2}} \mathbf{)}\end{array}$ & $\begin{array}{c}\text { Edge-disbond } \\
\left.\text { rate } \mathbf{( m m}^{\mathbf{2}} \mathbf{/ d a y}\right)\end{array}$ & $\begin{array}{c}\text { 'Wet' locus } \\
\text { of failure }\end{array}$ & $\begin{array}{c}\mathbf{G}_{\mathbf{C}} \text { ('wet') } \\
\left(\mathbf{J} / \mathbf{m}^{\mathbf{2}}\right)\end{array}$ & $\begin{array}{c}\text { Toughness } \\
\text { retained (\%) }\end{array}$ \\
\hline Grit blast & 251 & 25 & 0.1 & Interfacial & $48( \pm 16)$ & 37 \\
\hline CAE & 411 & 300 & 0.73 & Cohesive & $84( \pm 19)$ & 65 \\
\hline PAA & 566 & $\sim 0$ & 0 & Interfacial & $55( \pm 25)$ & 42 \\
\hline CAA & 566 & $\sim 0$ & 0 & Cohesive & $95( \pm 28)$ & 73 \\
\hline
\end{tabular}


2-layer specimen

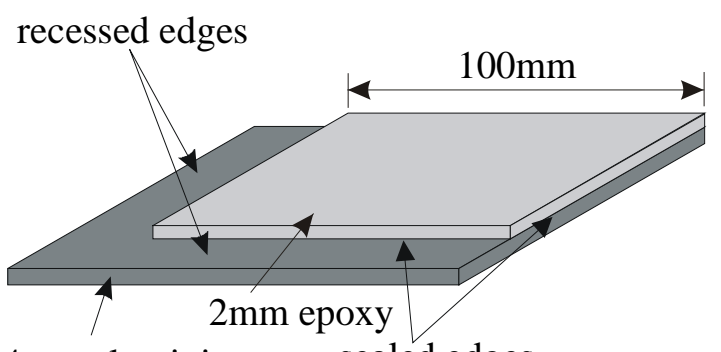

$4 \mathrm{~mm}$ aluminium sealed edges

\section{3-layer specimen}

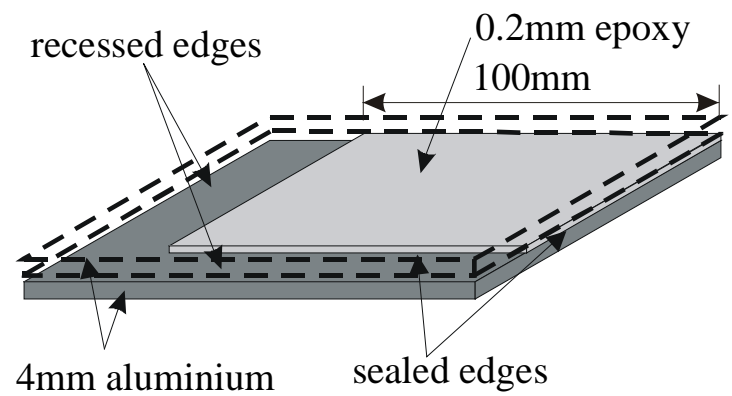

Figure 1 Two and three-layer specimen design.

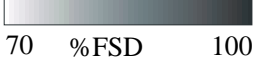

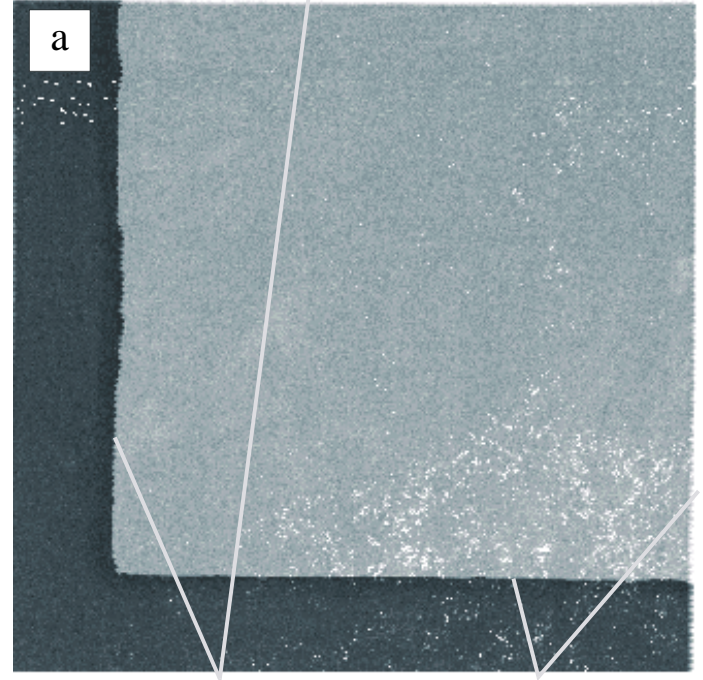

sealed edges

unsealed edges

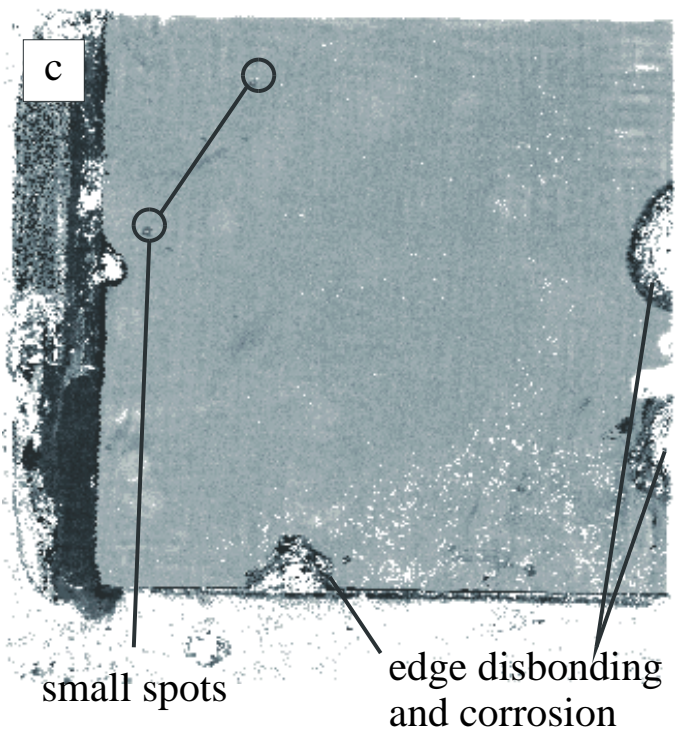

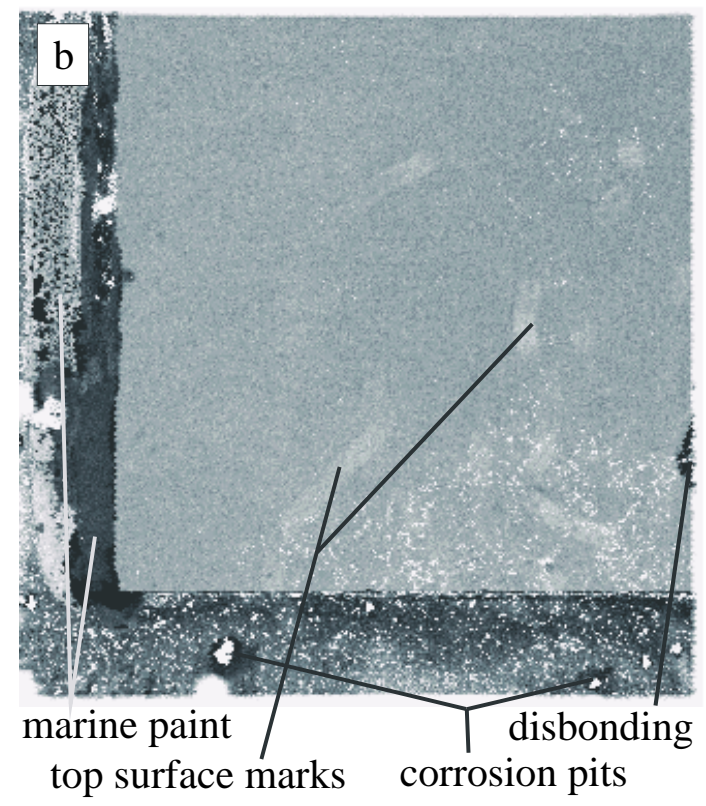

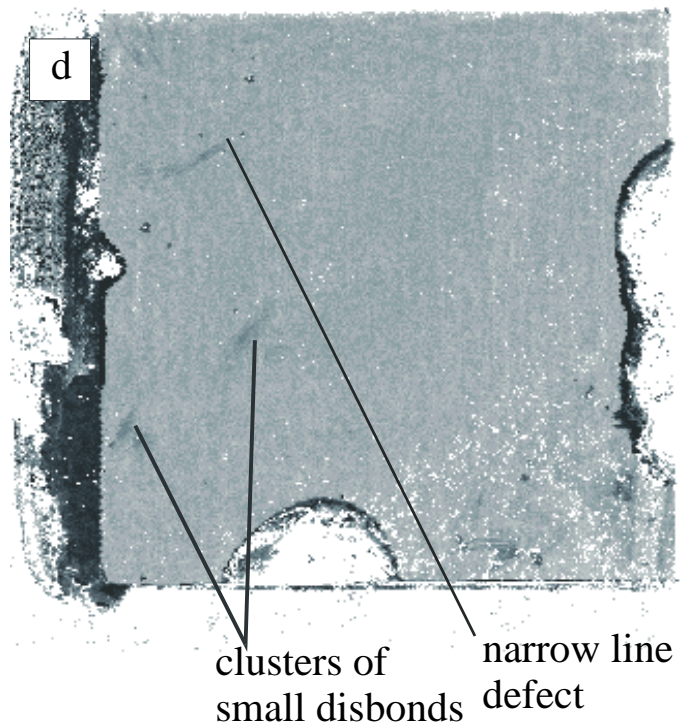

Figure 2 Normal-incidence scans from two-layer grit-blast specimens after exposure for: (a) 0 days (b) 27 days (c) 67 days (d) 117 days exposed to water at $50^{\circ} \mathrm{C}$. 

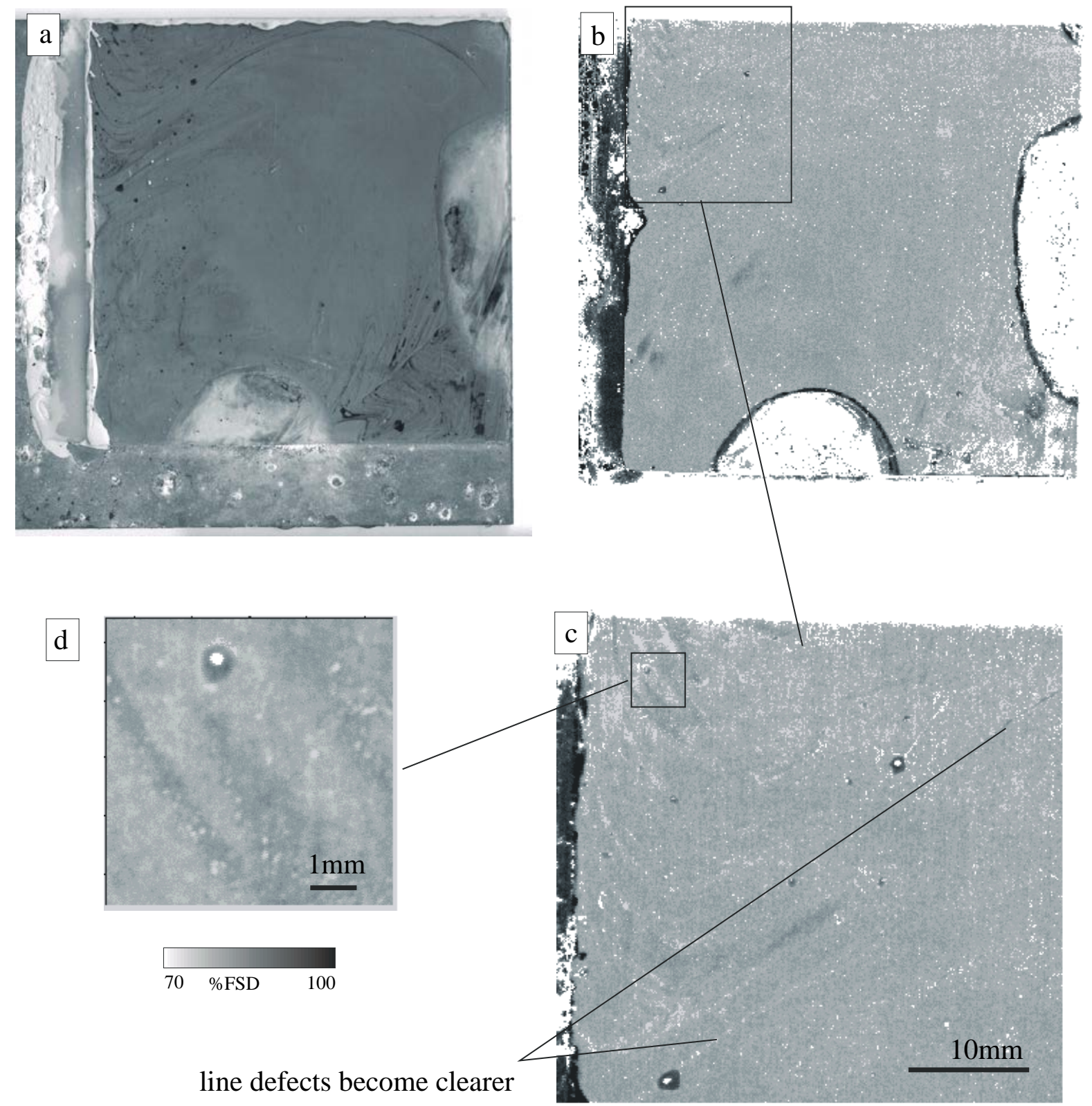

Figure 3 Two-layer grit-blast specimen after 223 days exposed to water at $50^{\circ} \mathrm{C}$ : (a) photograph; (b) ultrasonic scan at $0.25 \mathrm{~mm}$ scan pitch; (c) ultrasonic scan at $0.1 \mathrm{~mm}$ scan pitch; (d) ultrasonic scan at $0.025 \mathrm{~mm}$ scan pitch. 
(a)

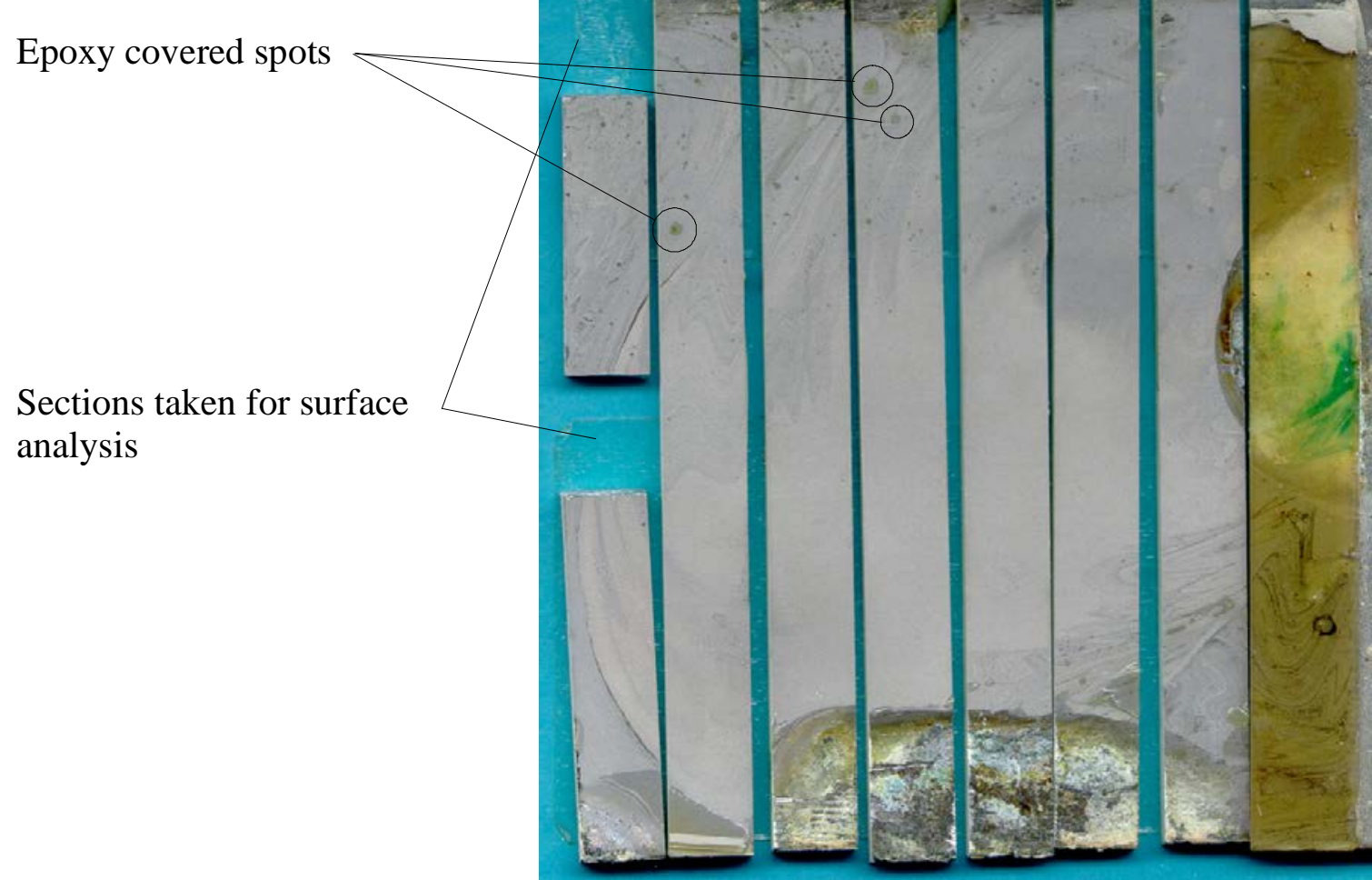

(b)

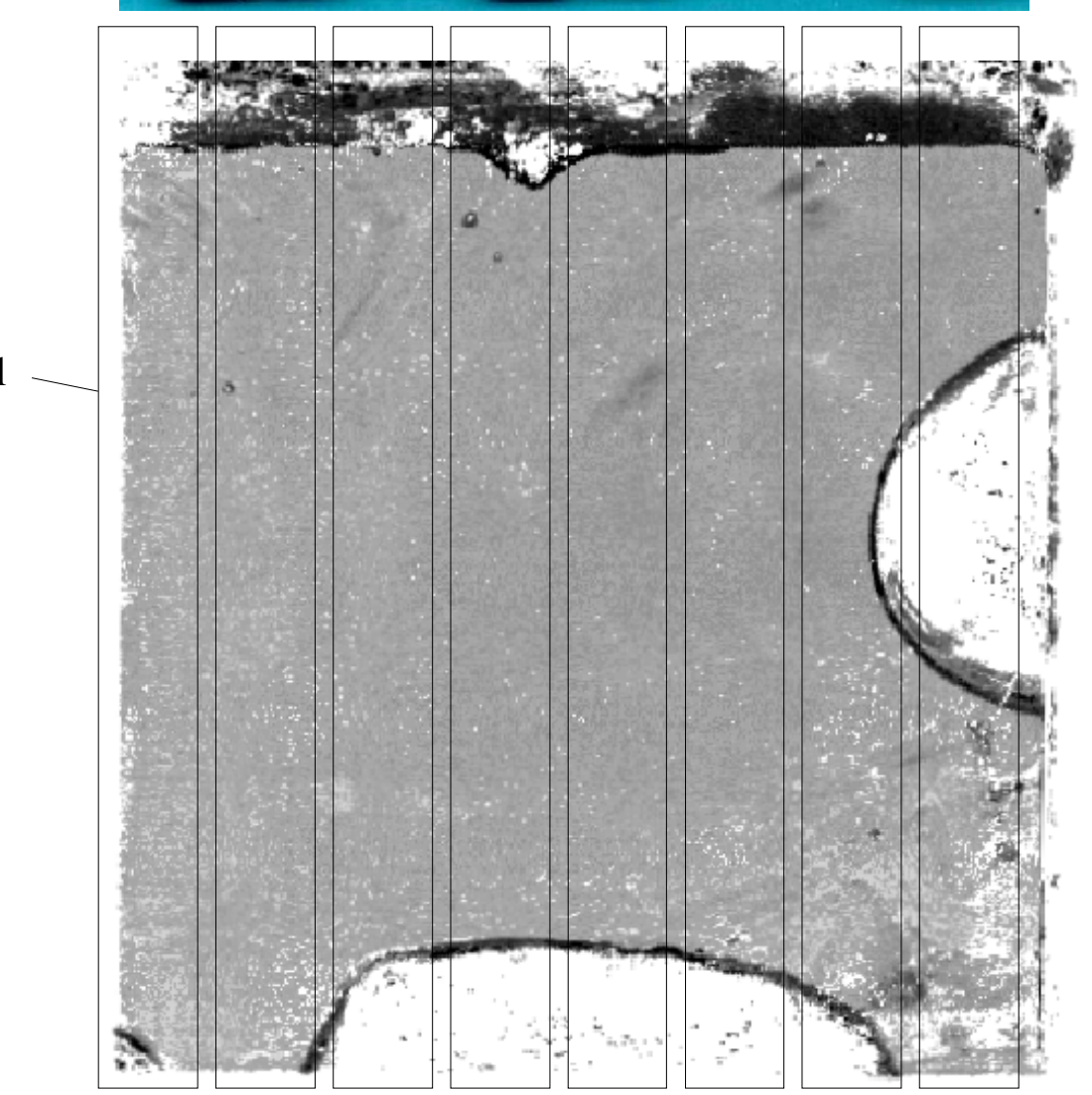

Figure 4 Two-layer grit-blast specimen after 223 days in water: (a) failure surface; (b) normalincidence ultrasonic scan. 
(a)

(b)

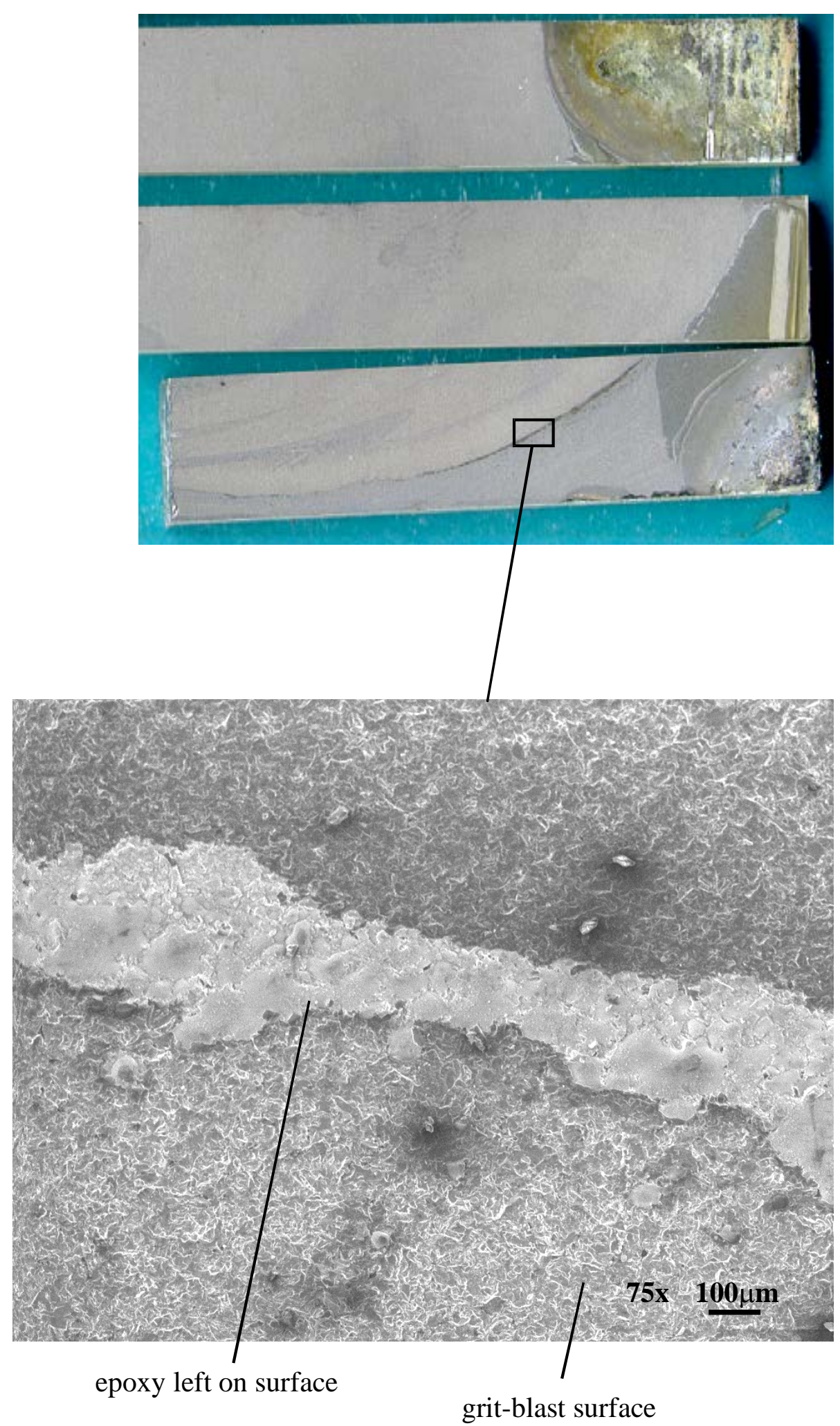

Figure 5 Failure surface from two-layer grit-blast specimen: (a) photograph; (b) scanning electron micrograph. 
Page 31

$\mathrm{a}$
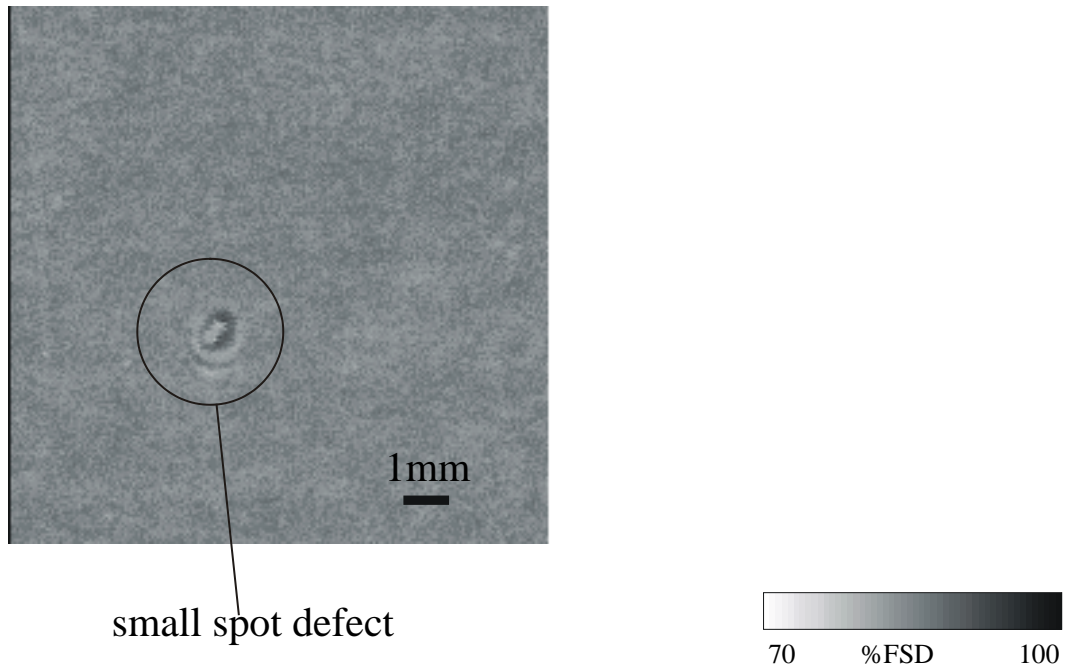

$\mathrm{b}$

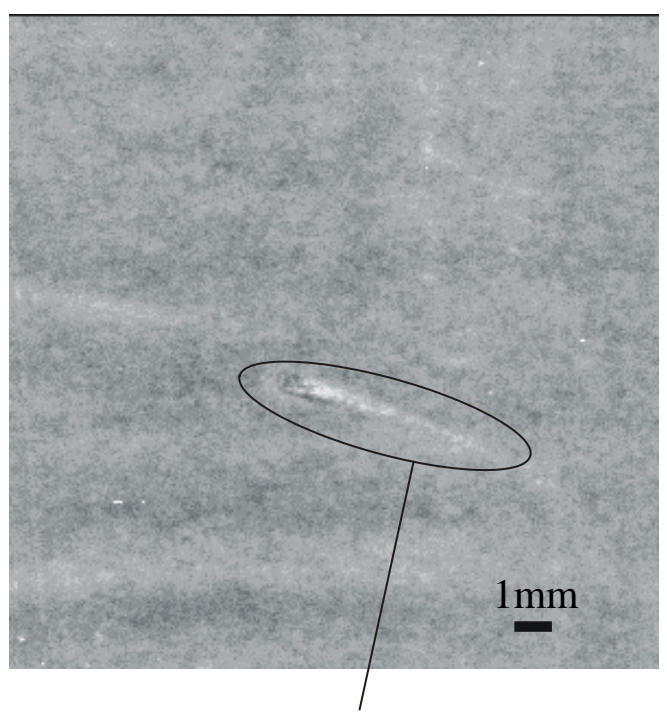

narrow line defect

Figure 6 Normal-incidence ultrasonic high-resolution scans of (a) a small spot micro-defect taken from a CAE specimen after 154 days in water at $50^{\circ} \mathrm{C}$ and (b) a narrow line micro-defect from the same CAE specimen after 194 days in water at $50^{\circ} \mathrm{C}$. 
(a)

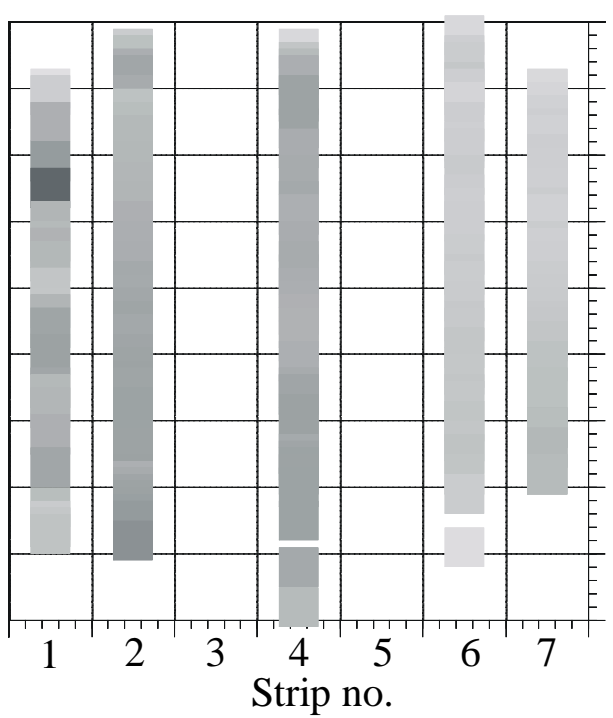

(b)

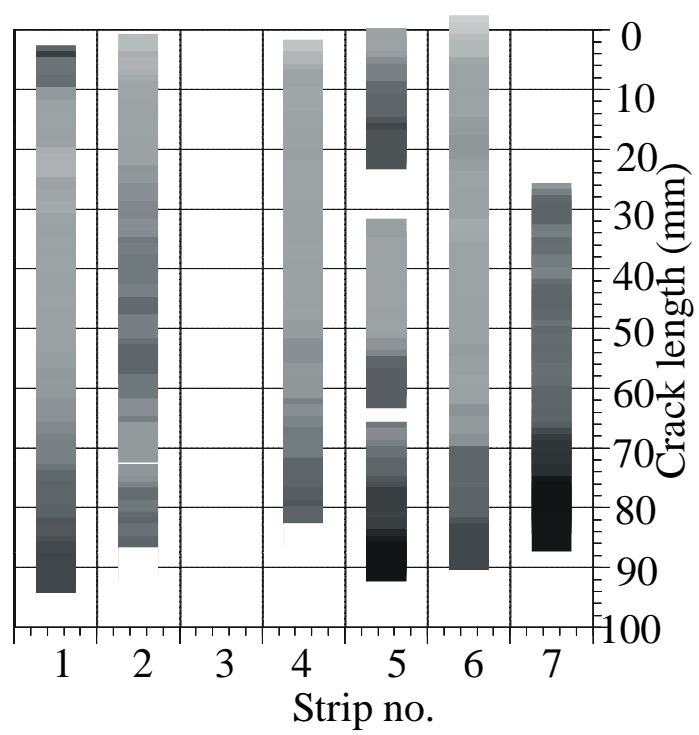

(c)

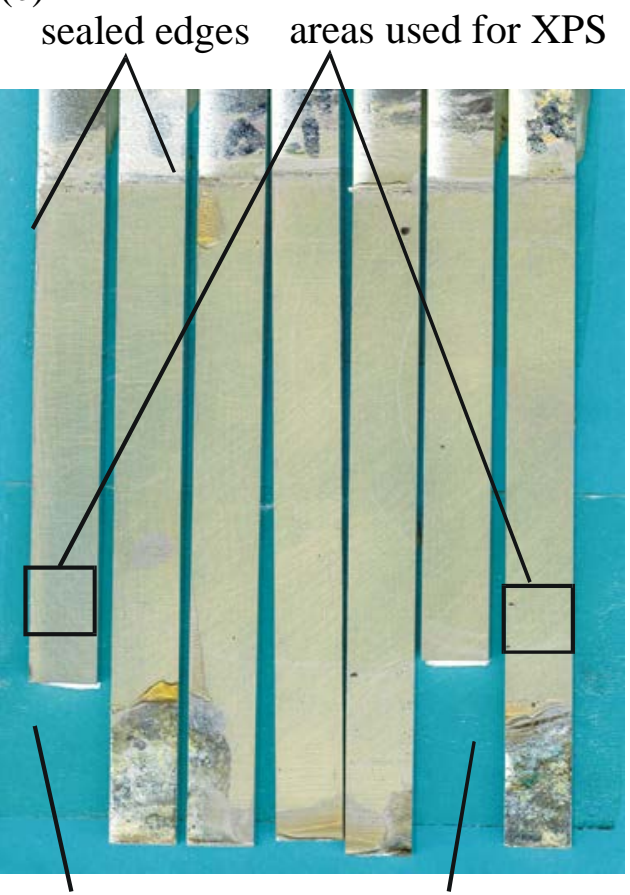

Sections taken for surface analysis (d)

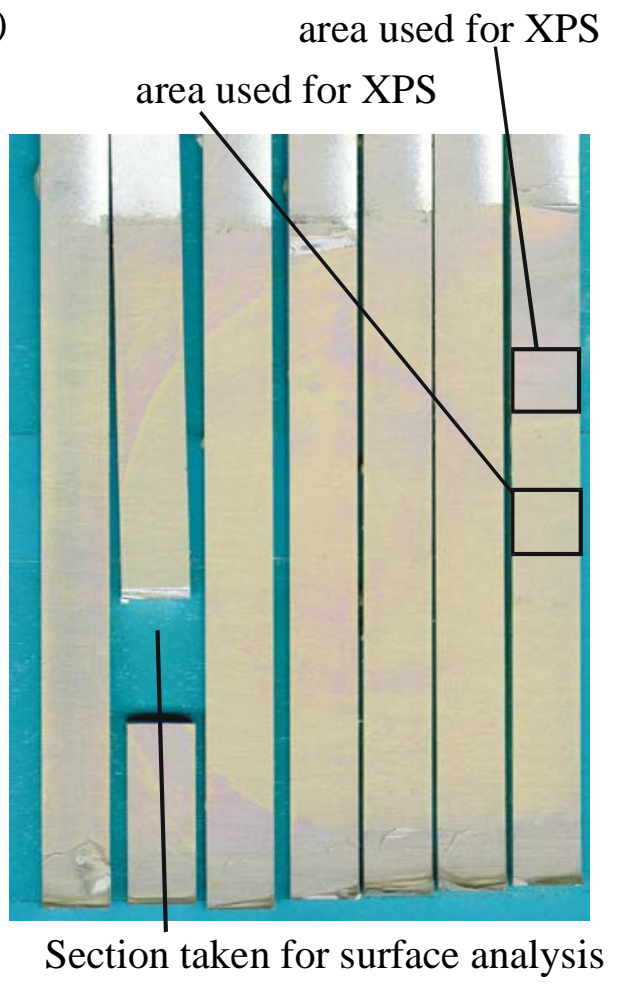

Figure $7 \quad$ Mechanical test results from the two-layer PAA specimens. Fracture toughness map from: (a) specimen after 393 days in water at $50^{\circ} \mathrm{C}$; (b) control 'dry' specimen. (c) photograph of the failure surface corresponding to (a); (d) photograph of the failure surface corresponding to (b). (The bottom and right-hand edges were the unsealed edges.) 

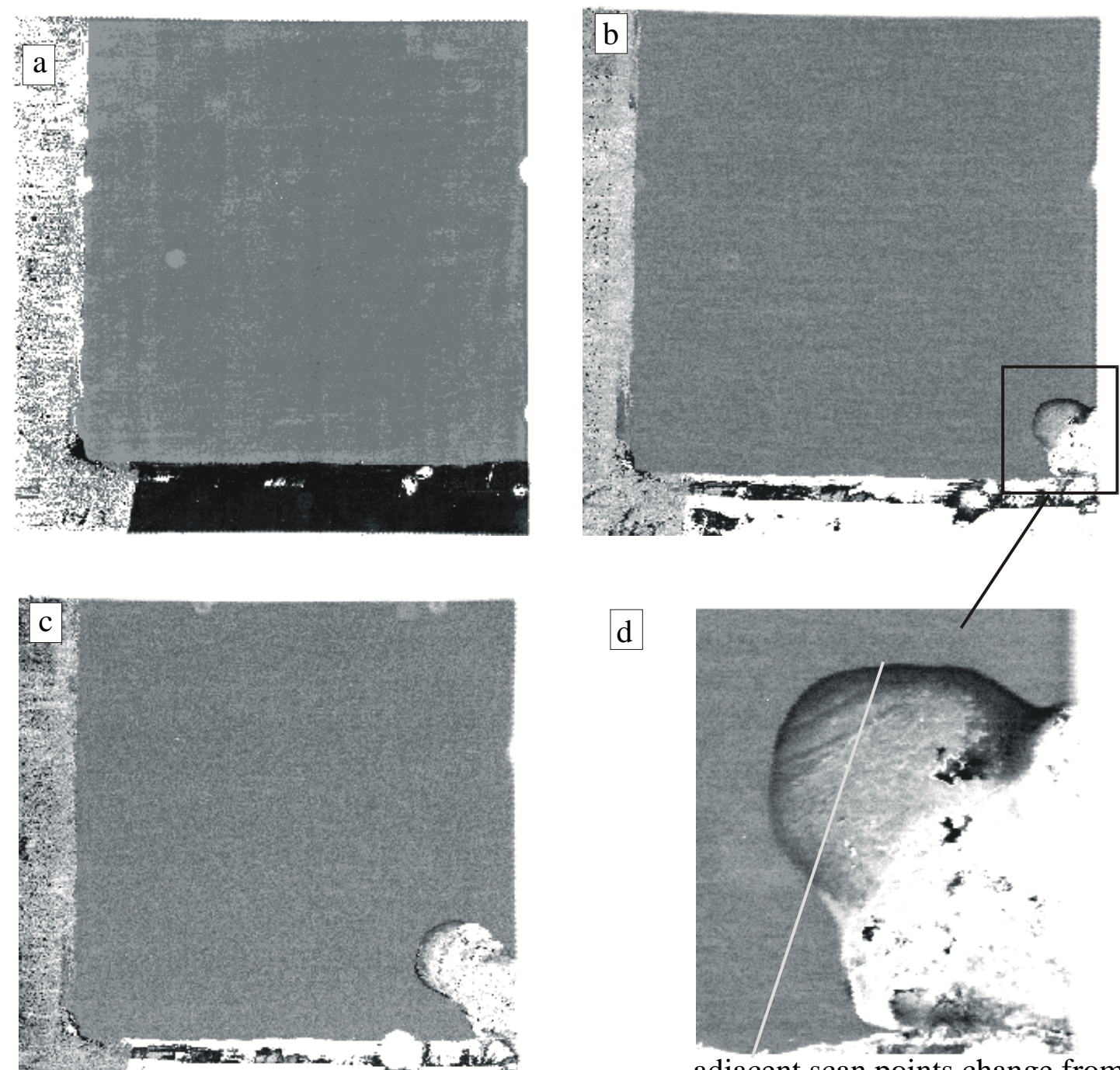
adjacent scan points change from bond to disbond

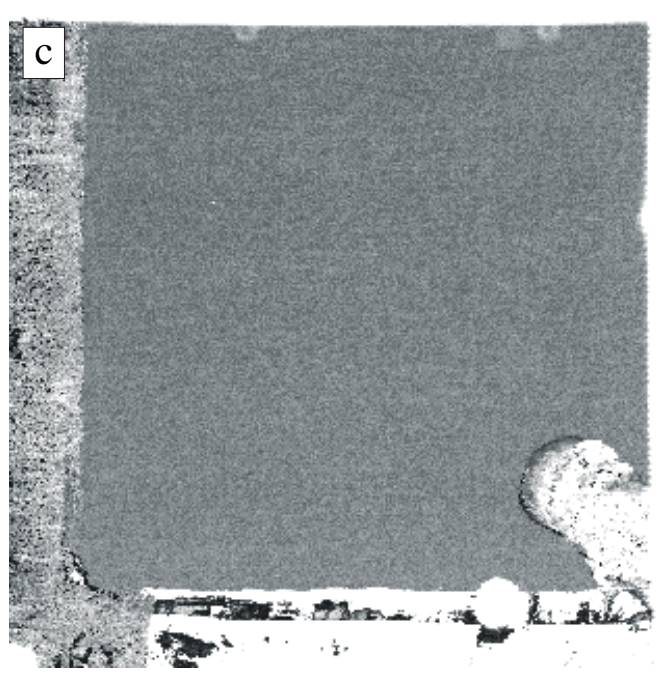

$\mathrm{f}$
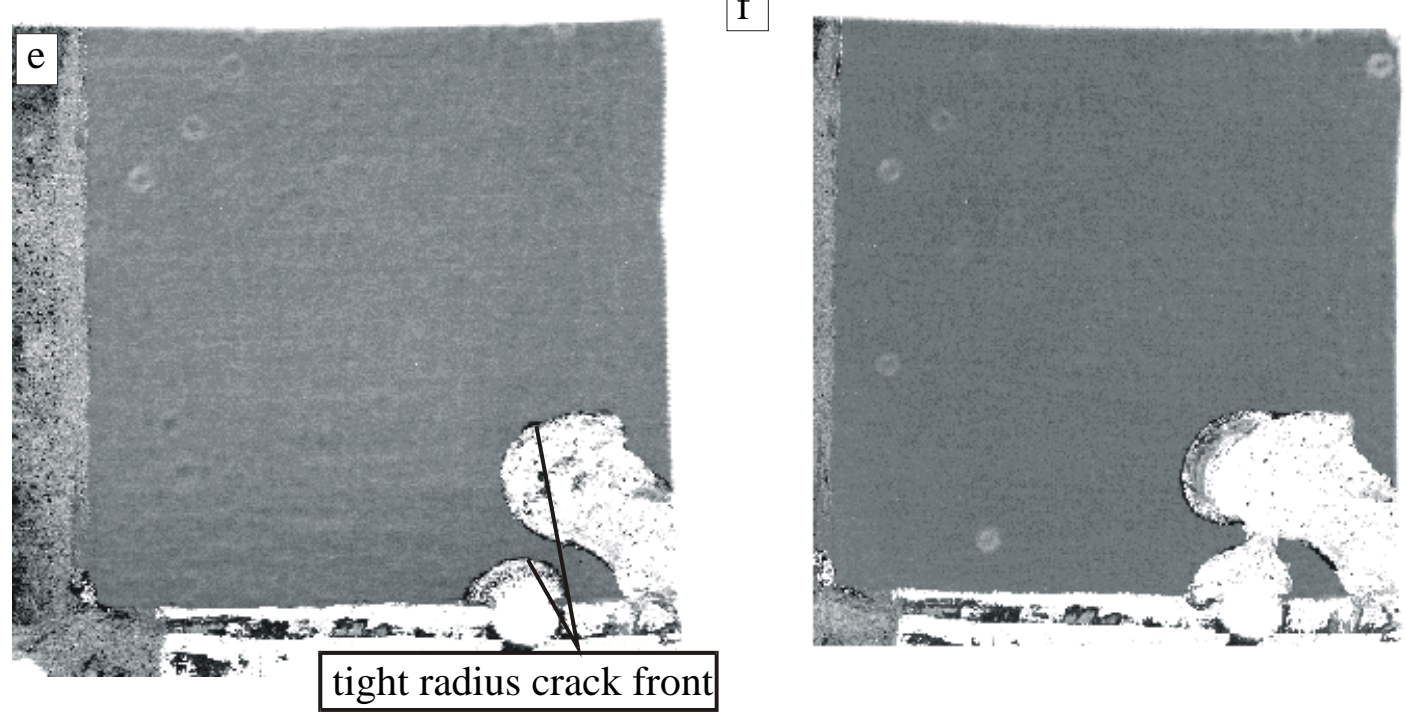

Figure $8 \quad$ Normal-incidence ultrasonic scans from the two-layer CAA specimen after: (a) 0; (b) 103; (c) 175; (e) 309; (f) 465 days in water at $50^{\circ} \mathrm{C}$; (d) high resolution scan of an edge-disbond in (b). 


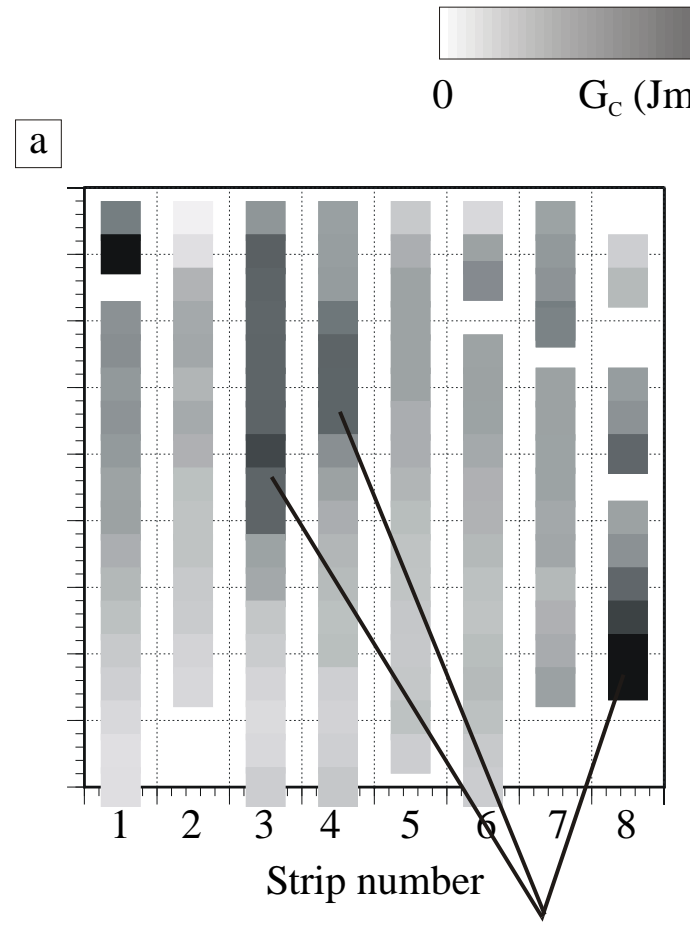

$$
0 \quad \mathrm{G}_{\mathrm{C}}\left(\mathrm{Jm}^{-2}\right) \quad 150
$$

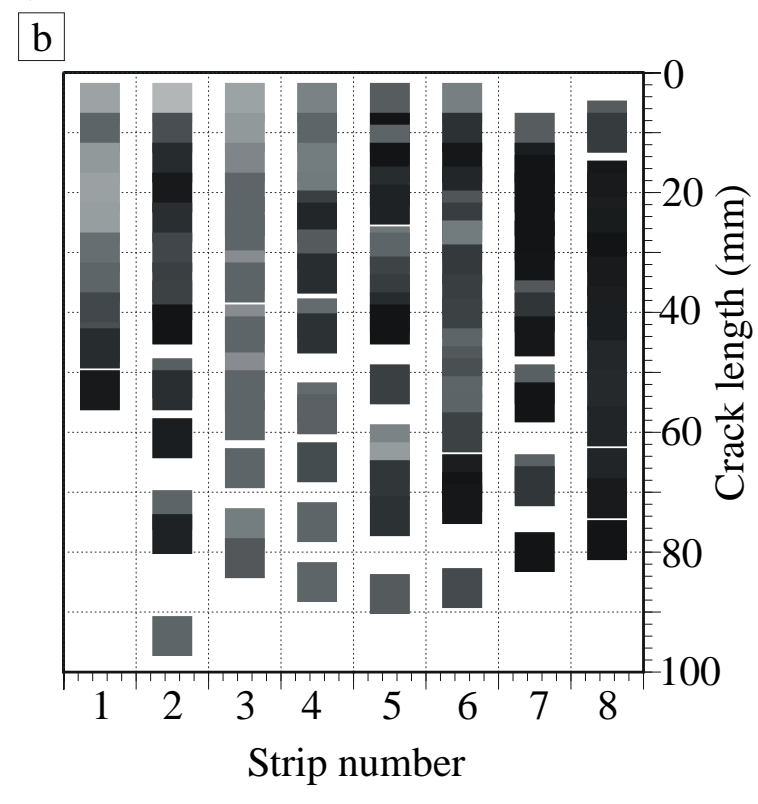

areas of cohesive failure
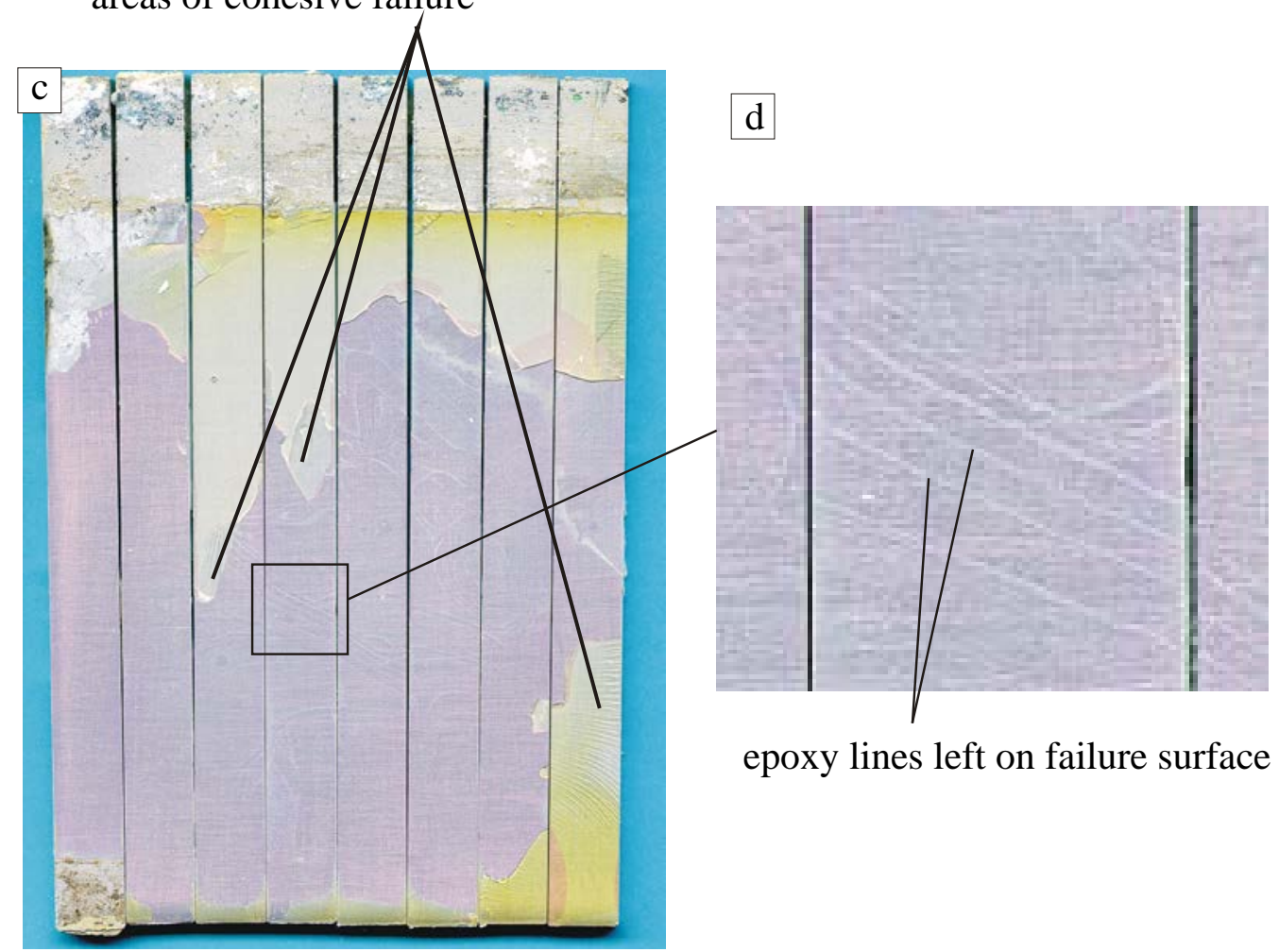

epoxy lines left on failure surface

Figure 9 Mechanical test results from three-layer PAA specimens. Fracture toughness map from: (a) specimen after 566 days in water; (b) control specimen. (c) failure surface corresponding to (a); (d) expanded view of section from (c) showing lines of epoxy on failure surface. 
Page 35
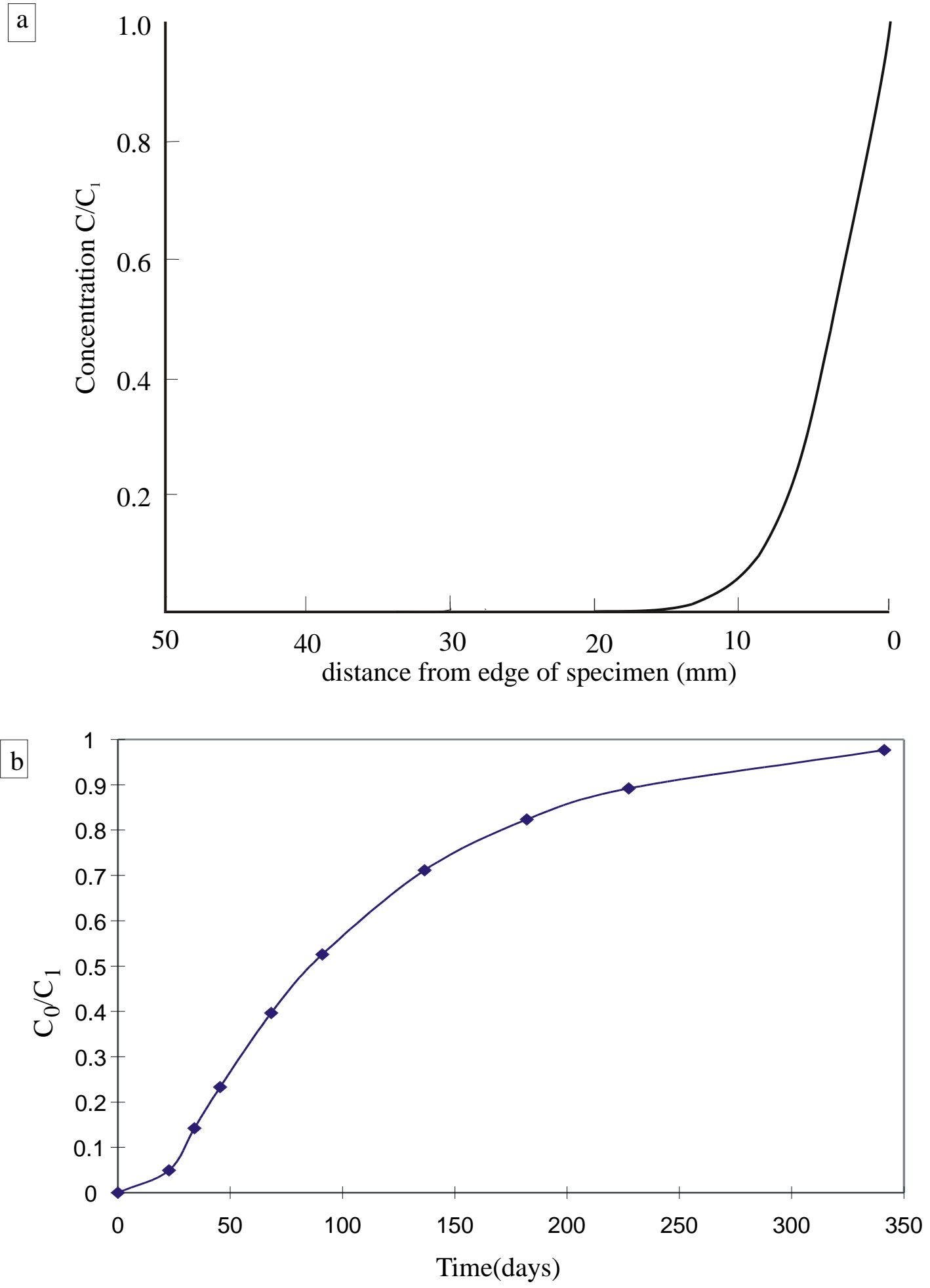

Figure 10 Predicted water concentration in the specimens as a fraction of saturation for: (a) in the epoxy layer of a three-layer specimen as a function of spatial position after 700 days in water at $50^{\circ} \mathrm{C}$; and (b) at the aluminum/epoxy interface of a two-layer specimen as a function of time in water at $50^{\circ} \mathrm{C}$. 